\title{
Spectral Properties of Random Schrödinger Operators with Unbounded Potentials
}

\author{
Y.A. Gordon ${ }^{1}$, V. Jakšić ${ }^{2 \star}$, S. Molčanov ${ }^{1}$, and B. Simon ${ }^{3 \star \star}$ \\ ${ }^{1}$ Department of Mathematics and Mechanics, Moscow State University, Moscow 119808, \\ Russia \\ ${ }^{2}$ Department of Mathematics, University of Toronto, Toronto, M5S-1A1, Canada \\ ${ }^{3}$ Division of Physics, Mathematics and Astronomy, California Institute of Technology, \\ Pasadena, CA 91125, USA
}

Received May 25, 1992

Abstract. We investigate spectral properties of random Schrödinger operators $H_{\omega}=-\Delta+\xi_{n}(\omega)\left(1+|n|^{\alpha}\right)$ acting on $l^{2}\left(Z^{d}\right)$, where $\xi_{n}$ are independent random variables uniformly distributed on $[0,1]$.

\section{Introduction}

It is already a part of folklore that multiplicative perturbations of the Anderson model show rather "unusual" spectral behavior. The basic paradigm is the discrete Schrödinger operator on $l^{2}\left(Z^{1}\right)$,

$$
\begin{aligned}
H_{\omega} u(n) & =2 u(n)-u(n+1)-u(n-1)+V_{\omega}(n) u(n), \\
V_{\omega}(n) & =\lambda \xi_{n}(\omega)|n|^{\alpha},
\end{aligned}
$$

where $\xi_{n}(\omega)$ are independent random variables with a bounded, compactly supported density $r(x)$, and $\lambda$ is a parameter. For $\alpha<0$ the above model has been extensively studied in $[5,7,8,18]$ and their main results can be summarized as follows (note that for $\alpha<0, V_{\omega}(n) \rightarrow 0$ as $|n| \rightarrow \infty$ and thus $\sigma_{\text {ess }}\left(H_{\omega}\right)=[0,4]$ ).

Theorem. With probability 1:

(i) For $-1 / 2<\alpha<0$, the spectrum in $[0,4]$ is pure point with eigenfunctions decaying as $\exp \left(-C|n|^{1+2 \alpha}\right)$.

(ii) For $\alpha<-1 / 2$, the spectrum in $[0,4]$ is purely absolutely continuous.

(iii) For $\alpha=-1 / 2$ and $\lambda$ large, the spectrum in $[0,4]$ is pure point with polynomially decaying eigenfunctions, while for $\lambda$ small $H_{\omega}$ will have some singular continuous spectrum.

\footnotetext{
* Research partially supported by a Sloan Doctoral Dissertation Fellowship and NSERC under grant OGP-0007901

$\star \star$ Research partially supported by NSF grant DMS-9101716
} 
For $\alpha>0,|n|^{\alpha} \rightarrow \infty$, but this does not imply that the spectrum is necessarily discrete: If $r(x)$ does not vanish in some neighborhood of $0, \xi_{n}$ can get arbitrarily small with positive probability and thus eventually compensate for the growth of $|n|^{\alpha}$ within infinitely many sites. That in turn can lead to nontrivial spectral behavior. Consider the simplest case when $\xi_{n}$ are independent random variables uniformly distributed on $[0,1]$ : It was shown in [9] that for a.e. $\omega, H_{\omega}$ will have a discrete spectrum if and only if $\alpha>1$. Furthermore, if $1 / k \geqq \alpha>1 /(k+1)$, $\sigma_{\text {ess }}\left(H_{\omega}\right)=\left[a_{k}, \infty\right)$, where $a_{k}$ is a strictly decreasing nonrandom sequence of positive numbers, $\sigma\left(H_{\omega}\right)=\sigma_{\mathrm{pp}}\left(H_{\omega}\right)$, and the eigenfunctions decay superexponentially. Thus, while for $\alpha<0$ the essential spectrum is always $[0,4]$ with a transition in its nature at $\alpha_{c}=-1 / 2$, for $\alpha>0$ the essential spectrum is always pure point but its end-point is piecewise constant function of the parameter $\alpha(!)$. In this paper we are interested in obtaining the multidimensional analog of the above results when $\alpha>0$, namely we will study the operator

$$
H_{\omega}=H_{0}+\xi_{n}(\omega)\left(1+|n|^{\alpha}\right), \quad \alpha>0,
$$

acting on $l^{2}\left(Z^{d}\right)$. In (1.1), $|n|=\left(\sum n_{i}^{2}\right)^{1 / 2}, \xi_{n}(\omega)$ are independent random variables uniformly distributed on $[0,1]$, and

$$
H_{0} \phi(n)=\sum_{|n-m|_{+}=1} \phi(n)-\phi(m), \quad\left\|H_{0}\right\|=4 d,
$$

where $|n|_{+}=\sum\left|n_{i}\right|$. We view $\xi_{n}(\omega)$ as a random field on $\bigotimes_{n \in Z^{d}}[0,1]=\Omega$, and denote by $P$ the corresponding probability measure, and by $\mathbf{E}$ the mathematical expectation on $\Omega$. Before stating our main results, we introduce some notation. For $X \subset Z^{d}$ denote by $C(X)$ the set of all $\phi \in l^{2}\left(Z^{d}\right)$ with support in $X$. Let $\mathscr{D}$ be the form associated to $H_{0}$,

$$
\mathscr{D}(\phi)=\sum_{\substack{(n, m) \\|n-m|_{+}=1}}|\phi(m)-\phi(n)|^{2},
$$

where $\langle n, m\rangle$ reminds one that each pair appears in the summation only once. Denote

$$
\Lambda(X)=\inf _{\substack{\|\phi\|=1 \\ \phi \in C(X)}} \mathscr{D}(\phi) .
$$

If $\# X<\infty, \Lambda(X)$ is the smallest eigenvalue of the spectral problem $H_{0} \phi=\lambda \phi$, $\phi(n)=0$ if $n \in Z^{d} \backslash X$. A path between points $n, m \in Z^{d}$ is a sequence of sites

$$
\tau=\left(n_{1}, n_{2}, \ldots, n_{k}\right), \quad n_{1}=n, \quad n_{k}=m, \quad\left|n_{j+1}-n_{j}\right|_{+}=1 .
$$

Set $X \subset Z^{d}$ is connected if any two points in $X$ can be connected with a path which lies within $X$. Following [10], we say that a set $A_{k} \subset Z^{d}$ is a $k$-animal (or just animal) if $A_{k}$ is connected and \# $A_{k}=k$. Modulo translation, there are only finitely many animals of any given size $k$. Let

$$
a_{k}=\inf _{A_{k}} \Lambda\left(A_{k}\right) \text {. }
$$

It is obvious that $a_{k}$ is a strictly decreasing sequence of positive numbers. The animals for which the infimum in (1.5) is attained we will denote by $A_{T, k}$ and call the tamed animals. As we will see later, taming the animals (namely, obtaining control over $a_{k}, A_{T, k}$ ) is not an easy task at all. 
In the sequel, $f(x) \sim g(x)$ stands for $\lim _{x \rightarrow \infty} f(x) / g(x)=1$, and $\tau_{d}$ for the volume of a unit ball in $R^{d}$. With the above notation, our main results are stated as follows.

Theorem 1.1. $H_{\omega}$ has a discrete spectrum $P$-a.s. if and only if $\alpha>d$. Furthermore, if $N_{\omega}(E)$ denotes the number of eigenvalues of $H_{\omega}$ which are less than $E$, we have that for $\alpha>d$ and for a.e. $\omega$

$$
N_{\omega}(E) \sim \frac{\tau_{d}}{\alpha-d} \cdot E^{d / \alpha} \text { as } E \rightarrow \infty
$$

Theorem 1.2. If $d / k \geqq \alpha>d /(k+1)$ for positive integer $k$, for a.e. $\omega$ we have

(i) $\sigma\left(H_{\omega}\right)=\sigma_{\mathrm{pp}}\left(H_{\omega}\right)$ and eigenfunctions of $H_{\omega}$ decay at least exponentially,

(ii) $\sigma_{\mathrm{ess}}\left(H_{\omega}\right)=\left[a_{k}, \infty\right)$,

(iii) $\# \sigma_{\text {disc }}\left(H_{\omega}\right)<\infty$.

Theorem 1.3. Let $\lambda_{D}$ be the lowest eigenvalue of a Dirichlet Laplacian of a unit ball in $R^{d}$. Then

$$
a_{k} \sim k^{-d / 2} \cdot \lambda_{D} \quad \text { as } k \rightarrow \infty .
$$

Remark 1. Only affecting the values of constants, we can suppose that the random variables $\xi_{n}$ have common nonnegative absolutely continuous density which is of the form $x^{\beta}$ on $[0, \delta)$ for some $\beta, \delta>0$. On the other hand if $\xi_{n}$ are uniformly distributed on $[-1,1]$, Theorem 1.1 remains valid (with appropriate reformulation of (1.6)). Part (i) of Theorem 1.2 also holds, while part (ii) has to be replaced with $\sigma\left(H_{\omega}\right)=(-\infty, \infty)$.

Remark 2. By the exponential decay of the eigenfunctions we mean that the eigenfunction of $H_{\omega}$ corresponded to an eigenvalue $E$ satisfies

$$
\left|\phi_{\omega, E}(n)\right| \leqq C_{\omega, E} \exp (-a|n|)
$$

for some fixed $a>0$. Our argument can be easily modified to show that eigenfunctions decay as

$$
\left|\phi_{\omega, E}(n)\right| \leqq C_{\omega, E} \exp (-a|n| \cdot \ln |n|) .
$$

The constant $\lambda_{D}$, introduced in Theorem 1.3, coincides with the smallest zero of the Bessel function $J_{d / 2-1}$. Thus $\lambda_{D}=2.4048 \ldots$ for $d=2, \lambda_{D}=\pi$ for $d=3$, and $\lambda_{D} \sim d / 2$ for $d$ large.

Remark 3. We will give two proofs that the spectrum of $H_{\omega}$ is pure point. The first one is rather simple and is based on the recent results on localization obtained in $[14,15]$. The first proof does not yield the satisfactory decay of the eigenfunctions, nor can its strategy be used to investigate the discrete spectrum. The second proof, although more complicated and relying heavily on the particular structure of our potential, yields immediately (1.7) and part (iii) of Theorem 1.2.

For the model (1.1) when $\alpha \leqq d$, one can also investigate the natural analog of the integrated density of states. Let $C_{L}=\left\{n: \max \left|n_{i}\right| \leqq L\right\}$ be the box of side $L$ centered at the origin. The form on $C\left(C_{L}\right)$ (recall (1.3))

$$
\mathscr{D}(\phi)+\left(V_{\omega} \phi, \phi\right)
$$


yields a finite matrix (it is just a restriction of $H_{\omega}$ to $C_{L}$ with Dirichlet boundary condition) whose number of eigenvalues which are less than $E$ we denote by $N_{\omega, L}(E)$. Set $a_{0}=\infty$.

\section{Theorem 1.4.}

(i) If $d / k>\alpha>d /(k+1)$ and $E \in\left(a_{j}, a_{j-1}\right), 1 \leqq j \leqq k$, then

$$
\lim _{L \rightarrow \infty} \frac{N_{\omega, L}(E)}{L^{d-j \alpha}}=N_{j}(E)
$$

exists for a.e. $\omega$ and is a non-random function.

(ii) If $\alpha=d / k$ and $E \in\left(a_{j}, a_{j-1}\right), 1 \leqq j<k$, (1.8) is valid. If $E \in\left(a_{k}, a_{k-1}\right)$ then

$$
\lim _{L \rightarrow \infty} \frac{N_{\omega, L}(E)}{\ln L}=N_{k}(E)
$$

exists for a.e. $\omega$ and is a non-random function.

In both cases, $N_{j}(E)$ is a continuous function on $\left(a_{j}, a_{j-1}\right)$, and

$$
N_{j}(E) \sim C_{j}\left(E-a_{j}\right)^{j} \quad \text { as } E \rightarrow a_{j} .
$$

The constants $C_{j}$ are of combinatorial nature.

The continuous analog of the model (1.1) is discussed in [11].

The paper is organized as follows. In Chapter 2 we prove Theorem 1.1, part (ii) of Theorem 1.2, and give a simple proof that $\sigma\left(H_{\omega}\right)=\sigma_{\mathrm{pp}}\left(H_{\omega}\right) P$-a.s. In Chapter 3 we prove Theorem 1.3 and in Chapter 4 we finish the proof of Theorem 1.2. In Chapter 5 we prove Theorem 1.4. Finally, in the Appendix we prove the result (announced in [16]) which has been used in the simple proof of localization.

\section{On the Discrete and Essential Spectrum}

2.1. Proof of Theorem 1.1. A sufficient and necessary condition for $H_{0}+V_{\omega}(n)$ to have discrete spectrum is that

$$
\left|V_{\omega}(n)\right| \rightarrow \infty \quad \text { as }|n| \rightarrow \infty .
$$

Let $c>0$ be fixed and denote

$$
A_{n}^{c}=\left\{\omega: \xi_{n}(\omega) \cdot\left(1+|n|^{\alpha}\right)<c\right\} .
$$

$P\left(A_{n}^{c}\right)=c /\left(1+|n|^{\alpha}\right)$ and $\sum_{n} P\left(A_{n}^{c}\right)$ converges if and only if $\alpha>d$. The BorelCantelli lemma implies that (2.1) is valid for a.e. $\omega$ iff $\alpha>d$, and the first part of Theorem 1.1 follows.

To calculate the eigenvalue asymptotics, first note that monotonicity of $N_{\omega}(E)$ implies that it is enough to consider the case when $E \rightarrow \infty$ as an integer. We proceed as follows. Denote by

$$
\chi_{E}^{ \pm}(n, \omega)= \begin{cases}1, & \text { if } \xi_{n}(\omega) \cdot\left(1+|n|^{\alpha}\right) \leqq E \pm 4 d ; \\ 0, & \text { otherwise } .\end{cases}
$$

and set $S_{E}^{ \pm}(\omega)=\sum_{n \in Z^{d}} \chi_{E}^{ \pm}(n, \omega)$. Because $\left\|H_{0}\right\|=4 d$, we have

$$
S_{E}^{-}(\omega) \leqq N_{\omega}(E) \leqq S_{E}^{+}(\omega) .
$$


Since

$$
E\left(S_{E}^{ \pm}\right)=\#\left\{n:\left(1+|n|^{\alpha}\right) \leqq E \pm 4 d\right\}+\sum_{n: 1+|n|^{\alpha}>E \pm 4 d} \frac{E \pm 4 d}{1+|n|^{\alpha}},
$$

one easily obtains

$$
\begin{aligned}
\lim _{E \rightarrow \infty} E^{-d / \alpha} \mathbf{E}\left(S_{E}^{ \pm}\right) & =\tau_{d}+\int_{|x|>1} \frac{1}{|x|^{\alpha}} d x \\
& =\frac{\tau_{d}}{\alpha-d} .
\end{aligned}
$$

It remains to show that

$$
E^{-d / \alpha}\left(S_{E}^{ \pm}-\mathbf{E}\left(S_{E}^{ \pm}\right)\right) \rightarrow 0 \quad \text { as } E \rightarrow \infty P \text {-a.s. }
$$

First note that

$$
\begin{aligned}
\mathbf{E}\left(\left(S_{E}^{ \pm}\right)^{k}\right) \leqq & 2^{k-1} \cdot\left(\left(\#\left\{n:\left(1+|n|^{\alpha}\right)<E \pm 4 d\right\}\right)^{k}\right. \\
& \left.+\sum_{\substack{\left(n_{1}, \ldots, n_{k}\right) \\
n_{i}>E \pm 4 d}} \frac{E \pm 4 d}{\prod_{i=1}^{k}\left(1+\left|n_{i}\right|^{\alpha}\right)}\right)
\end{aligned}
$$

and thus $\lim \sup _{E \rightarrow \infty} E^{-d k / \alpha} \mathbf{E}\left(\left(S_{E}^{ \pm}\right)^{k}\right) \leqq C$, where $C$ is a uniform constant. Let $\eta_{E}^{ \pm}(n, \omega)=\chi_{E}^{ \pm}(n, \omega)-\mathbf{E}\left(\chi_{E}^{ \pm}(n, \omega)\right) . \eta_{E}^{ \pm}(n)$ is a sequence of independent (but not identically distributed) random variables satisfying $\left|\eta_{E}^{ \pm}(n)\right| \leqq 2, \mathbf{E}\left(\eta_{E}^{ \pm}(n)\right)=0$. We have

$$
\begin{aligned}
\mathbf{E}\left(\left(S_{E}^{ \pm}-E\left(S_{E}^{ \pm}\right)\right)^{2 k}\right) & =\mathbf{E}\left(\left(\sum_{n \in \mathbf{Z}^{d}} \eta_{E}^{ \pm}(n)\right)^{2 k}\right) \\
& \leqq C \cdot \mathbf{E}\left(\left(S_{E}^{ \pm}\right)^{k}\right) \\
& \leqq C \cdot E^{d k / \alpha}
\end{aligned}
$$

for a uniform constant $C$. The first inequality in (2.4) follows from simple combinatorics and observations that

$$
\#\left\{n: \eta_{\bar{E}}^{ \pm}(n, \omega) \neq 0\right\} \leqq S_{E}^{ \pm}, \quad \mathbf{E}\left(\sum_{n \in Z^{d}} \eta_{\bar{E}}^{ \pm}(n)\right)=0 .
$$

For $\varepsilon>0$, Chebyshev's inequality yields

$$
\begin{aligned}
P_{E}=P\left\{E^{-d / \alpha}\left|S_{E}^{ \pm}-\mathbf{E}\left(S_{E}^{ \pm}\right)\right|>\varepsilon\right\} & \leqq \frac{E^{-2 d k / \alpha} \mathbf{E}\left(\left(S_{E}^{ \pm}-\mathbf{E}\left(S_{E}^{ \pm}\right)\right)^{2 k}\right)}{\varepsilon^{2 k}} \\
& \leqq C \frac{E^{-d k / \alpha}}{\varepsilon^{2 k}}
\end{aligned}
$$

If $d k / \alpha>1, \sum_{E=4 d+1}^{\infty} P_{E}<\infty$, and the Borel-Cantelli Lemma yields (2.3).

2.2. On the Essential Spectrum. In this section we prove that for $d / k \geqq \alpha>$ $d /(k+1), \sigma_{\text {ess }}\left(H_{\omega}\right)=\left[a_{k}, \infty\right)$. 
Denote $I=4 k \cdot Z^{d}$, and decompose $Z^{d}=\bigcup_{n \in I} \mathscr{T}_{n}$, where $\mathscr{T}_{n}$ is the cube of volume $(4 k)^{d}$ centered at $n$. Let $A_{T}$ be an arbitrary tamed $k$-animal which contains 0 , and denote by $A_{T}^{n}$ its translation for the vector $n \in I$. To show that

$$
\sigma_{\text {ess }}\left(H_{\omega}\right) \supset\left[a_{k}, \infty\right) \quad P \text {-a.s. }
$$

it suffices (using Weyl's criterion and fact that essential spectrum is closed set) to construct for every rational $\lambda>0$ and for a.e. $\omega$ a sequence $\varphi_{i}$ satisfying

$$
\lim _{i \rightarrow \infty}\left\|\left(H_{\omega}-\lambda-a_{k}\right) \varphi_{i}\right\|=0 \text {. }
$$

Fix a sequence $b_{i} \rightarrow 0$, and denote for $n \in I$

$$
A_{n}^{b_{i}}=\left\{\omega: \lambda-b_{i}<V_{\omega}(x)<\lambda+b_{i} \text { for } x \in A_{T}^{n}\right\} .
$$

$P\left(A_{n}^{b_{i}}\right) \geqq C b_{i} /\left(1+|n|^{\alpha}\right)^{k}$ for a suitable uniform constant $C$, and thus $\sum_{n \in I} P\left(A_{n}^{b_{2}}\right)$ diverges. The Borel-Cantelli lemma and diagonal argument implies that there exist with probability 1 a strictly increasing ( $\omega$-dependent) sequence $n_{i}$ such that

$$
\left|V_{\omega}(n)-\lambda\right|<b_{i} \text { for } n \in A_{T}^{n_{i}} .
$$

If $\varphi_{0}$ is eigenfunction corresponded to $a_{k}$, set $\varphi_{0}$ to be zero outside $A_{T}$ and denote $\varphi_{i}(n)=\varphi_{0}\left(n-n_{i}\right)$. It is trivial to check that (2.6) is satisfied and (2.5) follows. It remains to show that

$$
\inf \sigma_{\text {ess }}\left(H_{\omega}\right) \geqq a_{k} \quad P \text {-a.s. }
$$

Let $B(n, l)=\left\{m ;|n-m|_{+} \leqq l\right\}$. We will over and over again make use of the following

Lemma 2.1. For $\gamma>0$ let

$$
B_{n}=\left\{\omega \text { : in the box } B\left(n,|n|^{\gamma}\right) \text { there exists } k+1 \text { points } n_{i} \text { such that } V_{\omega}\left(n_{i}\right) \leqq\left|n_{i}\right|^{\gamma}\right\} \text {. }
$$

Then, for $\gamma$ small enough, with probability 1 , only finitely many events $B_{n}$ take place.

Proof. It is easy to show that for $\gamma$ small enough

$$
P\left(B_{n}\right) \leqq C \cdot|n|^{(k+1)(d \gamma+\gamma-\alpha)} \leqq C|n|^{-d-\varepsilon},
$$

where $C$ is a uniform constant. Thus, for such $\gamma, \sum_{n} P\left(B_{n}\right)<\infty$ and the BorelCantelli lemma yields the statement.

The discrete version of Persson's theorem ([4], Theorem 3.12) states

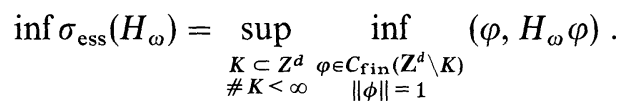

Let $K=K(\omega)$ be a box, centered at 0 , of large enough side so that outside $K$ no event $B_{n}$ takes place for a.e. $\omega$. Let

$$
R=\inf _{n \notin K}|n|^{\gamma},
$$

and let

$$
A_{\omega}=\left\{n \in Z^{d} \backslash K: V_{\omega}(n)<1\right\} .
$$


For a.e. $\omega, A_{\omega}$ is a disjoint union of animals of the size $\# \leqq k$. Let $B=Z^{d} \backslash\left(K \cup A_{\omega}\right)$, and for $\phi \in C_{\text {fin }}\left(Z^{d} \backslash K\right),\|\phi\|=1$ denote

$$
\phi_{1}=\left\{\begin{array}{ll}
\phi(n), & \text { if } n \in A_{\omega} ; \\
0, & \text { otherwise; }
\end{array} \quad \phi_{2}= \begin{cases}\phi(n) & \text { if } n \in B_{\omega} \\
0, & \text { otherwise }\end{cases}\right.
$$

Obviously,

$$
\left(\phi_{1}, \phi_{2}\right)=0, \quad\left\|\phi_{1}\right\|^{2}+\left\|\phi_{2}\right\|^{2}=1
$$

For $R>a_{k}$ we have

$$
\begin{aligned}
\left(H_{\omega} \phi, \phi\right) & =\left(H_{\omega} \phi_{1}, \phi_{1}\right)+\left(H_{\omega} \phi_{2}, \phi_{2}\right)+2 \operatorname{Re}\left(H_{\omega} \phi_{1}, \phi_{2}\right) \\
& \geqq a_{k}\left\|\phi_{1}\right\|^{2}+R\left\|\phi_{2}\right\|^{2}+2 \operatorname{Re}\left(H_{\omega} \phi_{1}, \phi_{2}\right) \\
& \geqq a_{k}+\left(R-a_{k}\right)\left\|\phi_{2}\right\|^{2}-2\left\|H_{0}\right\| \cdot\left\|\phi_{1}\right\| \cdot\left\|\phi_{2}\right\| \\
& \geqq a_{k}-\frac{16 d^{2}}{R-a_{k}} .
\end{aligned}
$$

Consequently

$$
\inf \sigma_{\text {ess }}\left(H_{\omega}\right) \geqq a_{k}-\frac{16 d^{2}}{R-a_{k}} \quad P \text {-a.s. }
$$

By taking $K$ big enough, $R$ can be made arbitrarily large and (2.7) follows.

2.3. A Simple Proof of Localization. We first fix some notation. Let $\partial X$ be defined as

$$
\partial X=\left\{n \in X:\left(\exists m \in Z^{d} \backslash X\right)|m-n|_{+}=1\right\} .
$$

For $X, Y \in Z^{d}$, let

$$
d(X, Y)=\min \left\{|n-m|_{+}: n \in X, m \in Y\right\} .
$$

The matrix elements of the resolvent of $H=H_{0}+V$ we denote by

$$
R(n, m ; z)=\left(\delta_{n},(H-z)^{-1} \delta_{m}\right) .
$$

The following (deterministic) result, which we will prove in the Appendix, has been announced in [16].

Theorem 2.2. Suppose that we have ascending sequence of connected sets in $Z^{d}$

$$
\Gamma_{1}^{-} \subset \Gamma_{1}^{+} \subset \Gamma_{2}^{-} \subset \Gamma_{2}^{+} \subset \ldots \subset \Gamma_{n}^{-} \subset \Gamma_{n}^{+} \subset \ldots
$$

such that

$$
\# \Gamma_{n}^{ \pm}=O\left(D_{n}^{d}\right) \quad \# \partial \Gamma_{n}^{ \pm}=O\left(D_{n}^{d-1}\right), \quad d_{n}=d\left(\Gamma_{n}^{-}, \Gamma_{n}^{+}\right),
$$

where $D_{n} \rightarrow \infty$ is a sequence of integers satisfying $D_{n} \leqq c^{n}$, for some $c$. Let $V$ be a potential satisfying

$$
V(m) \geqq C\left(D_{n}^{d-1} n^{1+\delta}\right)^{1 / d_{n}} \quad \text { if } m \in \Gamma_{n}^{+} \backslash \Gamma_{n}^{-},
$$


for some $C, \delta>0$. Then, for every $n \in Z^{d}$ and for a.e. $E \in R$ (with respect to the Lebesgue measure)

$$
\limsup _{\varepsilon \rightarrow 0} \sum_{m \in Z^{d}}|R(n, m ; E+i \varepsilon)|^{2}<\infty .
$$

The above theorem is a by-product of some recent results [14-16] on the localization in one dimension. On the intuitive level $[15,19]$, high-potential walls in $\Gamma_{n}^{+} \backslash \Gamma_{n}^{-}$are under conditions (2.10), (2.11) effective in stopping tunneling at large distances. An immediate consequence of (2.12) is that $\sigma_{\mathrm{ac}}(H)=\emptyset$.

To see how model (1.1) fits in the above picture we proceed as follows. Let $C_{k}$, $C_{k}^{\prime}$ be boxes of the sides $2^{k}-\left[2^{k \gamma-1}\right], 2^{k}+\left[2^{k \gamma-1}\right]$ where $\gamma$ is chosen as in (2.8). It is easy to show (using Lemma 2.1) that for a.e. $\omega$ there exists connected sets $\Gamma_{k}^{-}(\omega)$, $\Gamma_{k}^{+}(\omega)$, such that for $k>k_{0}(\omega)$

$$
C_{k} \subset \Gamma_{k}^{-}(\omega) \subset \Gamma_{k}^{+}(\omega) \subset C_{k}^{\prime}, \quad d_{k}=d\left(\Gamma_{k}^{+}(\omega), \Gamma_{k}^{-}(\omega)\right) \geqq C_{1} 2^{k \gamma},
$$

and that $V_{\omega}(m)>C_{2} 2^{k \gamma}$ for $m \in \Gamma_{k}^{+}(\omega) \backslash \Gamma_{k}^{-}(\omega)$. Thus, (2.10), (2.11) are obviously satisfied, and we have that for a.e. $\omega$ and a.e. $E$ and for every $n \in Z^{d}$

$$
\limsup _{\varepsilon \rightarrow 0} \sum_{m \in Z^{d}}\left|R_{\omega}(n, m ; E+i \varepsilon)\right|^{2}<\infty .
$$

Equation (2.13) and the Simon-Wolff theorem $[2,20]$ yields that $\sigma\left(H_{\omega}\right)=\sigma_{\mathrm{pp}}\left(H_{\omega}\right)$ for a.e. $\omega$.

Using Theorem 2.2 we get an easy proof that for the non-stationary model (1.1) (and for many others with unbounded potential) we have pure point spectrum. On the other hand, by their very nature, the theorems of the above type [16] cannot be used to prove exponential decay of eigenfunctions or to analyse the discrete spectrum. For that, one has to rely more heavily on the particular structure of our potential, and we will do it in Chapter 4.

\section{Taming the Animals}

This chapter is devoted to the proof of Theorem 1.3. We start by introducing the continuous analog of (1.3). If $\Omega$ is a region in $R^{d}$, the Dirichlet Laplacian $-\Delta_{D}^{\Omega}$ is the unique self-adjoint operator whose quadratic form is given by the closure of

$$
D(u)=\int_{\Omega}|\nabla u|^{2} d x
$$

on $C_{0}^{\infty}(\Omega)$. In the sequel we will suppose that all regions under consideration have a piecewise smooth boundary. If $|\Omega|$, the Lebesgue measure of $\Omega$, is finite, $-\Delta_{D}^{\Omega}$ has a compact resolvent and its smallest eigenvalue is given by

$$
\Lambda(\Omega)=\inf _{\substack{u \in C_{0}^{\infty}(\Omega) \\\|u\|=1}} D(u) .
$$

The corresponding eigenfunction $u$ is a $C^{\infty}(\Omega)$ function satisfying

$$
-\Delta u(x)=\Lambda(\Omega) u(x) \text { for } x \in \Omega, \quad u(x)=0, \text { if } x \in \partial \Omega .
$$


It is also known that $u(x)>0$ if $x \in \Omega$. For positive $r$ let

$$
\lambda_{r}=\inf \{\Lambda(\Omega):|\Omega|=r\} \text {. }
$$

The infimum in (3.2) is achieved when $\Omega$ is a ball of volume $r$ [4], $\lambda_{1}=\lambda_{D}$ and $\lambda_{r}=\lambda_{D} / r^{2 / d}$. For notational simplicity, in the sequel we will suppose that $d=2$. We will prove

\section{Lemma 3.1.}

$$
a_{k} \leqq \frac{\lambda_{D}}{k} \cdot(1+O(1 / \sqrt{k}))
$$

\section{Lemma 3.2.}

$$
a_{k} \geqq \frac{\lambda_{D}}{k} \cdot \frac{1+O(1 / \sqrt{k})}{1+3 \cdot \# \partial A_{T, k} / k} .
$$

Lemma 3.3. If $A_{T, k}, k \geqq 1$ is a sequence of tamed $k$-animals, we have

$$
\lim _{k \rightarrow \infty} \frac{\# \partial A_{T, k}}{k}=0 \text {. }
$$

Theorem 1.3 is an immediate consequence of the above three lemmas. Their proofs are somewhat subtle and we devote to each a separate section.

3.1. Proof of Lemma 3.1. To avoid confusion, throughout the chapter we use Latin letters to denote elements of $L^{2}\left(R^{d}\right)$, and Greek letters for elements of $l^{2}\left(Z^{d}\right)$. By $\|\cdot\|_{\infty}$ we denote the norm on $R^{2}$ given by $\left\|\left(\varepsilon_{1}, \varepsilon_{2}\right)\right\|_{\infty}=\max \left\{\left|\varepsilon_{1}\right|,\left|\varepsilon_{2}\right|\right\}$. To any subset $X$ of $Z^{2}$ we correspond a region $X_{a}$ in $R^{2}$ whose closure is given by

$$
\bar{X}_{a}=\left\{x \in R^{2}: \sup _{y \in X}\|x-y\|_{\infty} \leqq a\right\} .
$$

If $A_{k}$ is an arbitrary animal, $A_{k, 1 / 2}$ the region given by (3.3) with $a=1 / 2$. For $\Lambda\left(A_{k, 1 / 2}\right)$ given by (3.1), we denote the corresponding eigenfunction by $u$ and set $u(x)=0$ if $x \notin A_{k, 1 / 2}$. To every $x \in F=\left\{x \in R^{2}:\|x\|_{\infty}<1 / 2\right\}$ we associate a function $\phi_{x}$ on $Z^{2}$ as $\phi_{x}(n)=u(n+x), n \in Z^{2}$. Obviously, $\phi_{x}(n) \neq 0$ iff $n \notin A_{k}$. Furthermore,

$$
\int_{F}\left\|\phi_{x}\right\|^{2} d x=\int_{F} \sum_{n} u(n+x)^{2} d x=\sum_{n} \int_{F} u(n+x)^{2} d x=\|u\|^{2} .
$$

Let $e_{1}=(1,0), e_{2}=(0,1)$. We have for $i=1,2$,

$$
\begin{aligned}
\int_{F} \sum_{n}\left(\phi_{x}\left(n+e_{i}\right)-\phi_{x}(n)\right)^{2} d x & =\sum_{n} \int_{F}\left(u\left(n+x+e_{i}\right)-u(n+x)\right)^{2} d x \\
& =\sum_{n} \int_{F}\left(\int_{0}^{1} \frac{\partial u\left(n+x+t e_{i}\right)}{\partial x_{i}} d t\right)^{2} d x \\
& \leqq \int_{0}^{1} \sum_{n} \int_{F}\left(\frac{\partial u\left(n+x+t e_{i}\right)}{\partial x_{i}}\right)^{2} d x d t \\
& =\int_{R^{2}}\left(\frac{\partial u(x)}{\partial x_{i}}\right)^{2} d x .
\end{aligned}
$$


Summing over $i$ we obtain

$$
\int_{F} \mathscr{D}\left(\phi_{x}\right) d x \leqq D(u),
$$

and consequently there exists at least one $x_{0} \in F$ such that

$$
\mathscr{D}\left(\phi_{x_{0}}\right) \leqq D(u)=\Lambda\left(A_{k, 1 / 2}\right)\|u\|^{2}=\Lambda\left(A_{k, 1 / 2}\right)\left\|\phi_{x_{0}}\right\|^{2} .
$$

It is immediate that

$$
a_{k} \leqq \Lambda\left(A_{k}\right) \leqq \Lambda\left(A_{k, 1 / 2}\right) .
$$

Let $a>1 / \sqrt{2}$ be arbitrary, and denote by $B(r)$ the ball of radius $r$ centered at 0 . For $k$ large enough let $S=B(\sqrt{k / \pi}-a) \cap Z^{2}$. We have

$$
\# S=\left|S_{1 / 2}\right| \leqq|B(\sqrt{k / \pi}-a+1 / \sqrt{2})|<|B(\sqrt{k / \pi})|=k,
$$

and so there exists a $k$-animal $A_{k}$ that contains $S$. We have

$$
A_{k, 1 / 2} \supset S_{1 / 2} \supset B(\sqrt{k / \pi}-a-1 / \sqrt{2}),
$$

and consequently

$$
\begin{aligned}
a_{k} \leqq \Lambda\left(A_{k, 1 / 2}\right) & \leqq \Lambda(B(\sqrt{k / \pi}-a-1 / \sqrt{2}) \\
& =\frac{\lambda_{D}}{\pi(\sqrt{k / \pi}-a-1 / \sqrt{2})^{2}} \\
& =\frac{\lambda_{D}}{k}(1+O(1 / \sqrt{k})) .
\end{aligned}
$$

The lemma follows.

3.2. Proof of Lemma 3.2. Let $A_{k} \subset Z^{2}$ be an arbitrary animal, and let $\phi$ be the normalized eigenfunction corresponding to $\Lambda\left(A_{k}\right)$. We extend $\phi$ to a continuous function $u: R^{2} \rightarrow R$ in the following way: $u(k)=\phi(k)$ if $k \in Z^{2}$, and on the each quadrant $Q_{i j}=\left\{\left(x_{1}, x_{2}\right): i \leqq x_{1} \leqq i+1, j \leqq x_{2} \leqq j+1\right\}, u$ is of the form

$$
u\left(x_{1}, x_{2}\right)=a_{i j}+b_{i j} x_{1}+c_{i j} x_{2}+d_{i j} x_{1} x_{2} .
$$

Such extension exists and is unique. We divide the rest of the argument into three steps.

Step 1. $D(u) \leqq \mathscr{D}(\phi)$. The change of variable $\varepsilon_{1}=x_{1}-i-1 / 2, \varepsilon_{2}=x_{2}-j-1 / 2$, transforms $Q_{i j}$ into the quadrant $Q=\left\{\left(\varepsilon_{1}, \varepsilon_{2}\right):\left|\varepsilon_{1}\right| \leqq 1 / 2,\left|\varepsilon_{2}\right| \leqq 1 / 2\right\}$, and $u(x)$ into the function

$$
U(\varepsilon)=h+a \varepsilon_{1}+b \varepsilon_{2}+c \varepsilon_{1} \varepsilon_{2},
$$

for suitable $i, j$-dependent constants $h, a, b, c$. We have

$$
\int_{Q_{i j}}\left(\frac{\partial u}{\partial x_{1}}\right)^{2} d x=\int_{Q}\left(\frac{\partial U}{\partial \varepsilon_{1}}\right) d \varepsilon=\int_{Q}\left(a+c \varepsilon_{2}\right)^{2} d \varepsilon=a^{2}+c^{2} / 12,
$$


and

$$
\begin{aligned}
\frac{1}{2}\left((u(i+1, j)-u(i, j))^{2}+(u(i+\right. & \left.1, j+1)-u(i, j+1))^{2}\right) \\
= & \frac{1}{2}\left((U(1 / 2,-1 / 2)-U(-1 / 2,-1 / 2))^{2}\right. \\
& \left.+(U(1 / 2,1 / 2)-U(-1 / 2,1 / 2))^{2}\right) \\
= & \frac{1}{2}\left((a-c / 2)^{2}+(a+c / 2)^{2}\right)=a^{2}+c^{2} / 4
\end{aligned}
$$

From (3.7), (3.8) we obtain, summing over $i, j$,

$$
\int_{R^{2}}\left(\frac{\partial u}{\partial x_{1}}\right)^{2} d x \leqq \sum_{i, j}(u(i+1, j)-u(i, j))^{2}
$$

and similarly

$$
\int_{R^{2}}\left(\frac{\partial u}{\partial x_{2}}\right)^{2} d x \leqq \sum_{i, j}(u(i, j+1)-u(i, j))^{2} .
$$

The result follows by adding (3.9), (3.10).

Step 2. $\|u\|^{2} \geqq 1-3 \Lambda\left(A_{k}\right) / 4$. First, note that

$$
\begin{aligned}
\int_{Q_{i, j}} u(x)^{2} d x & =\int_{Q}\left(h+a \varepsilon_{1}+b \varepsilon_{2}+c \varepsilon_{1} \varepsilon_{2}\right)^{2} d \varepsilon_{1} d \varepsilon_{2} \\
& =h^{2}+\left(a^{2}+b^{2}\right) / 12+c^{2} / 124 \\
& \geqq h^{2}=u(i+1 / 2, j+1 / 2)^{2} .
\end{aligned}
$$

Consequently,

$$
\|u\|^{2} \geqq \sum_{i, j} u(i+1 / 2, j+1 / 2)^{2} .
$$

Let $\hat{Z}^{2}$ be a dual lattice of $Z^{2}$, obtained by translating $Z^{2}$ by the vector $(1 / 2,1 / 2)$. Let $T: l^{2}\left(Z^{2}\right) \rightarrow l^{2}\left(\hat{Z}^{2}\right)$ be the averaging operator defined as

$$
T \psi(i+1 / 2, j+1 / 2)=\frac{1}{4} \sum_{ \pm, \pm} \psi(i+1 / 2 \pm 1 / 2, j+1 / 2 \pm 1 / 2) .
$$

From its very definition, $\left.u\right|_{\hat{Z}^{2}}=T \phi$, and

$$
\|u\| \geqq\|u\|_{\hat{Z}^{2}}\|=\| T \phi \| .
$$

The adjoint $T^{*}: l^{2}\left(\hat{Z}^{2}\right) \rightarrow l^{2}\left(Z^{2}\right)$ is given by

$$
T^{*} \eta(i, j)=\frac{1}{4} \sum_{ \pm, \pm} \eta(i \pm 1 / 2, j \pm 1 / 2)
$$

and we have $\|T\|=\left\|T^{*}\right\|$,

$$
\|u\| \geqq\left\|T^{*} T \phi\right\| .
$$


Let $B_{n}(a)$ be a ball of radius $a$ centered at $n$ (in the euclidean metric), and denote by $B(a)$ the $n$-independent number of integer points contained in $B_{n}(a)$. Let $K_{a}: l^{2}\left(Z^{2}\right) \rightarrow l^{2}\left(Z^{2}\right)$ be defined as

$$
K_{a} u(n)=\frac{1}{B(a)} \sum_{m \in B_{n}(a)} u(m)
$$

For example, $H_{0}=4\left(K_{1}-1\right)$. For any $k$ we have $\Lambda\left(A_{k}\right) \leqq 4, \phi \geqq 0$ on $A_{k}$, and consequently

$$
K_{1} \phi(n) \geqq\left(1-\Lambda\left(A_{k}\right) / 4\right) \phi(n), \quad K_{1}^{2} \phi(n) \geqq\left(1-\Lambda\left(A_{k}\right) / 4\right)^{2} \phi(n) .
$$

A direct calculation yields

$$
\begin{aligned}
& T^{*} T=\frac{1}{4} \mathbf{1}+\frac{1}{2} K_{1}+\frac{1}{4} K_{\sqrt{2}}, \\
& K_{\sqrt{2}}=2 K_{1}^{2}-\frac{1}{2} K_{2}-\frac{1}{2} \mathbf{1} .
\end{aligned}
$$

Thus, using (3.12) we obtain

$$
\|u\| \geqq\left\|T^{*} T \phi\right\| \geqq\left(T^{*} T \phi, \phi\right) \geqq \frac{1}{2}\left(1-\Lambda\left(A_{k}\right) / 4\right)+\frac{1}{2}\left(1-\Lambda\left(A_{k} / 4\right)\right)^{2},
$$

and consequently,

$$
\|u\|^{2} \geqq 1-3 \Lambda\left(A_{k}\right) / 4
$$

Step 3

$$
a_{k} \geqq \frac{\lambda_{D}}{k} \cdot \frac{1+O(1 / \sqrt{k})}{1+3 \cdot \# \partial A_{T, k} / k} .
$$

From the above two steps we obtain, if $\Lambda\left(A_{k}\right) \leqq 4 / 3$,

$$
\frac{D(u)}{\|u\|} \leqq \frac{\mathscr{D}(\phi)}{1-3 \Lambda\left(A_{k}\right) / 4}=\frac{\Lambda\left(A_{k}\right)}{1-3 \Lambda\left(A_{k}\right) / 4} .
$$

Let $A_{k, 1}$ be given by (3.3) with $a=1$. $u$ belongs to the quadratic form domain of the Dirichlet Laplacian in the region $A_{k, 1}$, and from (3.15) we get

$$
\Lambda\left(A_{k, 1}\right) \leqq \frac{\Lambda\left(A_{k}\right)}{1-3 \Lambda\left(A_{k}\right) / 4} .
$$

Consequently,

$$
\begin{aligned}
& \Lambda\left(A_{k}\right) \geqq \frac{\Lambda\left(A_{k, 1}\right)}{\left(1+3 \Lambda\left(A_{k, 1}\right) / 4\right)} \geqq \frac{\lambda_{D}\left|A_{k, 1}\right|^{-1}}{1+3 \lambda_{D}\left|A_{k, 1}\right|^{-1 / 4}} \\
&=\lambda_{D}\left|A_{k, 1}\right|^{-1}(1+O(1 / k)) .
\end{aligned}
$$

Equation (3.16) is trivially satisfied if $\Lambda\left(A_{k}\right)>4 / 3$, and so is true for all $k$. We also have $\left|A_{k, 1}\right| \leqq \# A_{k}+3 \cdot \# \partial A_{k}$ and consequently

$$
\Lambda\left(A_{k}\right) \geqq \frac{\lambda_{D}}{k+3 \cdot \# \partial A_{k}} \cdot(1+O(1 / k)) \geqq \frac{\lambda_{D}}{k} \cdot \frac{1+O(1 / \sqrt{k})}{k+3 \cdot \# \partial A_{k}} .
$$


Equation (3.17) is true for any $k$-animal, and in particular for any tamed $k$-animal $A_{T, k}$. The lemma follows.

3.3. Proof of Lemma 3.3. For. $X \subset Z$ denote

$$
\begin{gathered}
\partial_{n} X=\{m \in X: \text { there exists a path of length } \leqq n \text { starting at } m \\
\text { and ending at } \left.m^{\prime} \in Z^{2} \backslash X\right\} .
\end{gathered}
$$

Obviously, $\partial_{1} X=\partial X$. If $A_{T, k}, k>0$, is a chosen sequence of tamed animals, we will prove

$$
\lim _{k \rightarrow+\infty} \frac{\# \partial_{n} A_{T, k}}{k}=0
$$

The lemma follows by setting $n=1$ in (3.18). As before, $\phi$ denotes the normalized eigenfunction corresponded to $a_{k}=\Lambda\left(A_{T, k}\right)$. For $X \subset Z^{d}$ let

$$
\|\phi\|_{X}^{2}=\sum_{n \in X}|\phi(n)|^{2}
$$

Again, we split the proof into three steps.

Step 1. $\|\phi\|_{\partial_{n} A_{T, k}}^{2}=O\left(a_{k}\right)=O(1 / k)$.

If $m \in \partial_{n} A_{T, k}$, there exists a path of length $l \leqq n, m=m_{1}, m_{2}, \ldots, m_{l}$, $\left|m_{j-1}-m_{j}\right|_{+}=1, m_{l} \notin A_{T, k}$. We have

$$
\begin{gathered}
|\phi(m)| \leqq \sum_{j=1}^{l}\left|\phi\left(m_{j-1}\right)-\phi\left(m_{j}\right)\right|^{2}, \\
|\phi(m)|^{2} \leqq n \sum_{j=1}^{l}\left|\phi\left(m_{j-1}\right)-\phi\left(m_{j}\right)\right|^{2} .
\end{gathered}
$$

Any couple $\left(m, m^{\prime}\right),\left|m-m^{\prime}\right|_{+}=1$ can belong only to finitely many paths of length. $n$, the number depending only on $n$, and consequently there exists a uniform constant $C_{n}$ so that

$$
\sum_{m \in \partial_{n} A_{T, k}} \phi(k)^{2} \leqq C_{n} \sum_{\substack{\left\langle m^{\prime}, m\right\rangle \\\left|m^{\prime}-m\right|_{+}=1}}\left|\phi\left(m^{\prime}\right)-\phi(m)\right|^{2}=C_{n} \cdot a_{k}=O(1 / k) .
$$

Step 2. Denote $O_{k}^{n}=A_{T, k} \backslash \partial_{n} A_{T, k}$, and let $\psi(m)=\max \{0, \phi(m)-b / \sqrt{k}\}$ for $0<b<1$. Then

(a) $\mathscr{D}(\psi) \leqq \mathscr{D}(\phi)$.

(b) $\|\psi\| \geqq 1-b$.

(c) If $Z_{k}^{n}=O_{k}^{n} \cup\left\{m \in A_{T, k}: \psi(m)>0\right\}$ then $\#\left(Z_{k}^{n} \backslash O_{k}^{n}\right)=O(1)$ as $k \rightarrow \infty$.

The part (a) is trivial. To prove (b), let $\hat{\phi}(m)=\max \{\phi(m), b / \sqrt{k}\}$. Then

$$
\|\hat{\phi}\|_{\boldsymbol{A}_{T, k}}^{2}=\sum_{m \in A_{T, k}}|\hat{\phi}(m)|^{2} \leqq\|\phi\|^{2}=1, \quad \psi(m)=\hat{\phi}(m)-b / \sqrt{k},
$$

and the Minkowski inequality yields

$$
\|\psi\| \geqq\|\hat{\phi}\|_{A_{T, k}}-\left(\sum_{m \in A_{T, k}} b^{2} / k\right)^{1 / 2} \geqq 1-b .
$$


To prove (c), note that from Step 1 we have

$$
\#\left\{m \in \partial_{n} A_{T, k}: \phi(m)>b / \sqrt{k}\right\}=O(1) .
$$

On the other hand, $\psi(m)>0$ iff $\phi(m)>b / \sqrt{k}$ and (c) follows.

Step 3. If $\alpha_{n}=\lim \sup _{k \rightarrow \infty}\left(\# \partial_{n} A_{T, k}\right) / k$ then $\lim _{n \rightarrow \infty} \alpha_{n}=0$.

We first remark that $0 \leqq \alpha_{1} \leqq \alpha_{2} \leqq \ldots \leqq 1$, and consequently $\lim _{n \rightarrow \infty} \alpha_{n}=\alpha$ exists. Let $\theta=1-\alpha$ and suppose that $\theta<1$. Let $\rho \in(\theta, 1)$ be arbitrary, and denote

$$
\theta_{n}=\liminf _{k \rightarrow \infty} \frac{\# Z_{k}^{n}}{k}
$$

From Step 2 we have that

$$
\frac{\# Z_{k}^{n}}{k}=\frac{O(1)}{k}+1-\frac{\# \partial_{n} A_{T, k}}{k},
$$

and consequently $\theta_{n}=1-\alpha_{n} \leqq \rho$ for $n$ large enough. Step 2 also implies that for any $b>0$ and any $k_{0} \in \mathfrak{N}$, there exist $k, m, k>k_{0}, m<\rho k$, so that $\# A_{k}^{n}=m$ and

$$
\Lambda\left(Z_{k}^{n}\right) \leqq a_{k} /(1-b)^{2} .
$$

First, (3.20) implies that $\theta \geqq 1 / 4$. Otherwise, we can choose $\rho<1 / 4$ and derive from (3.17), (3.20)

$$
\begin{aligned}
\frac{\lambda_{D}}{4 m} \cdot(1+O(1 / m)) & \leqq a_{m} \leqq \Lambda\left(Z_{k}^{n}\right) \\
& \leqq \frac{a_{k}}{(1-b)^{2}} \leqq \frac{\lambda_{D}}{k(1-b)^{2}} \cdot(1+O(1 / \sqrt{k})),
\end{aligned}
$$

$m / k<\rho$, and so when $k \rightarrow \infty, m \rightarrow \infty$ as well, and we obtain $1 / 4 \leqq \rho(1-b)^{2}$ for all $b>0$, which contradicts the choice $\rho<1 / 4$.

Let $0<\varepsilon<1$ be chosen in such a way that $\rho / \theta<1 /(1-\varepsilon)$. For $n$ large enough, $\theta_{n}<\rho$ and consequently $\theta / \theta_{n} \geqq 1-\varepsilon$. There exists a sequence $k_{j} \rightarrow \infty$ so that

$$
\lim _{j \rightarrow \infty} \frac{\# \partial_{n} A_{T, k_{j}}}{k_{j}}=\alpha_{n} .
$$

On the other hand, from (3.19) we derive

$$
\begin{array}{r}
\limsup _{j \rightarrow \infty} \frac{\# \partial_{n+1} A_{T, k_{j}}}{k_{j}} \leqq \alpha_{n+1}<1, \\
\lim _{j \rightarrow \infty} \frac{\# O_{n}^{k_{j}}}{k_{j}}=\theta_{n}, \quad \liminf _{j \rightarrow \infty} \frac{\# O_{n+1}^{k_{j}}}{k_{j}} \geqq \theta_{n+1} \geqq \theta .
\end{array}
$$

Because $\partial O_{k}^{n}=O_{k}^{n} \backslash O_{k+1}^{n}$, we have

$$
\limsup _{j \rightarrow \infty} \frac{\# \partial O_{k_{j}}^{n}}{\# O_{k_{j}}^{n}} \leqq \frac{\theta_{n}-\theta}{\theta_{n}}<\varepsilon .
$$


Again, Step 2 (c) yields

$$
\limsup _{j \rightarrow \infty} \frac{\# \partial Z_{k_{j}}^{n}}{\# Z_{k_{j}}^{n}}<\varepsilon .
$$

For $j$ large enough, to any $k_{j}$ we correspond $m_{j}$ with $m_{j}<\rho k_{j}$ so that $\# Z_{k_{j}}^{n}=m_{j}$ and (3.20) is true. It follows from (3.17) that for any $\delta>0$ and large enough $j$

$$
\Lambda\left(Z_{k_{j}}^{n}\right) \geqq \frac{\lambda_{D}(1-\delta)}{m_{j}\left(1+3 \cdot \# \partial Z_{k_{j}}^{n} / m_{j}\right)} \geqq \frac{\lambda_{D}(1-\delta)}{\rho k_{j}(1+3 \varepsilon)} .
$$

On the other hand, from (12.1) and Lemma 12.1 we obtain

$$
\Lambda\left(Z_{k_{j}}^{n}\right) \leqq \frac{a_{k_{j}}}{(1-b)^{2}} \leqq \frac{\lambda_{D}}{k_{j}(1-b)^{2}} \cdot\left(1+O\left(1 / \sqrt{k_{j}}\right)\right)
$$

and consequently for a large $j$ we have

$$
\rho \geqq(1-b)^{2} \frac{1-\delta}{(1+3 \varepsilon)(1+\delta)} .
$$

The numbers $b, \varepsilon, \delta$ can be chosen to be arbitrarily small and so $\rho \geqq 1$, which contradicts the choice of $\rho$, namely that $\theta<\rho<1$. So, $\theta=1$, and for all $n, \alpha_{n}=0$. The lemma follows.

\section{On the Pure Point and the Discrete Spectrum}

The following simple consequences of Lemma 2.1 will be of essential importance below. Let $d / k \geqq \alpha>d /(k+1)$, and for suitable $\gamma$ let

$$
A_{\omega}=\left\{n: V_{\omega}(n) \leqq|n|^{\gamma}\right\} \text {. }
$$

According to Lemma 2.1, we have a decomposition

$$
A_{\omega}=\bigcup_{n \geqq 0} A(n, \omega), \quad A(n, \omega) \cap A(m, \omega)=\emptyset,
$$

for a.e. $\omega$. (In the sequel we drop the $\omega$ dependence whenever it is clear within the context.) $A(0)$ is a finite region chosen in such a way that for $n \notin A(0)$ no events $B_{n}$ take place (see (2.8)), and $A(n)$ are animals of size $\leqq k$. Let $\sim$ be a relation of equivalence on $\{A(n): n>0\}$ defined as

$$
\begin{gathered}
A(n) \sim A(m) \Leftrightarrow \exists n_{1}=n, n_{2}, \ldots, n_{l}=m \text { such that for } 1 \leqq i \leqq l-1, \\
d\left(A\left(n_{i}\right), A\left(n_{i+1}\right)\right) \leqq C \max \left\{|s|^{\gamma},\left|s^{\prime}\right|^{\gamma}: s \in A\left(n_{i}\right), s^{\prime} \in A\left(n_{i+1}\right)\right\} .
\end{gathered}
$$

We order classes of equivalence by their distance from the origin (viewing classes as subsets of $Z^{d}$ ), ordering arbitrarily the equidistant ones. Let $G^{(i)}$ be a union of the elements of the $i^{\text {th }}$ class. By taking $C$ small enough, it is easy to show that $\# G^{(i)} \leqq k$ (otherwise some event $B_{n}$ happens for $n \notin A(0)$ ) and that

$$
d\left(G^{(i)}, G^{(k)}\right)>C \max \left\{|s|^{\gamma},\left|s^{\prime}\right|^{\gamma}: s \in G^{(i)}, s^{\prime} \in G^{(k)}\right\} .
$$

We will refer to $G^{(i)}$ as a connected group of animals. 
The point of the above is the following: if we think about $G^{(i)}$ as potential wells, then, for typical $\omega$ and outside a finite ( $\omega$-dependent) set, these wells have no more than $k$ points and are separated by high and long potential barriers. The probabilistic framework differs now from the one in Sect. 2.3, where we were interested only in potential wells encircling each other successively and thus preventing tunneling. By taking into account the structure of $G^{(i)}$, one should be able to obtain better control of the resolvent matrix elements, and that will be our main concern below. We will prove

Theorem 4.1. Let $I=\left(0, E_{0}\right)$. Then for every $E_{0}>0$ there exists $a>0$ such that for every $n \in Z^{d}$ and for a.e. $\omega \in \Omega$ and a.e. $E \in I$ (with respect to the Lebesgue measure)

$$
\sup _{0<\varepsilon<1}\left|R_{\omega}(n, m ; E+i \varepsilon)\right| \leqq C_{n, \omega, E} \exp (-a|m|) .
$$

The above theorem is certainly expected on intuitive grounds. (Actually, more is expected, namely that $|m|$ can be replaced with $|m|^{\beta}$ for some $\beta>1$, although we will not try to prove that.) Simple modification of our argument can improve decay in (4.2) by a factor $\ln |m|$.) Once proven, Theorem 4.1 yields part (i) of Theorem 1.2 by the well-known argument of Simon-Wolff [20] or Delyon-Levy-Souillard [6] (see also [2]).

The rest of this chapter is devoted to the proof of Theorem 4.1. Part (iii) of Theorem 1.2 is a simple by-product of the analysis below, and necessary modifications in the argument are outlined in Sect. 4.2.

The following decomposition of a probability space $\Omega=\bigotimes_{n \in Z^{d}} I_{n}, I_{n}=[0,1]$, will be useful. If $\gamma>0$ is the constant from Lemma 2.1, writing

$$
I_{n}=I_{n}^{0} \cup I_{n}^{1}, \quad I_{n}^{0}=\left[0,|n|^{\gamma} /\left(1+|n|^{\alpha}\right)\right], \quad I_{n}^{1}=\left[|n|^{\gamma} /\left(1+|n|^{\alpha}\right), 1\right],
$$

we obtain the decomposition

$$
\Omega=\bigcup_{c} \Omega_{c}
$$

indexed by all possible sequences of 0 and 1's. One simple consequence of discussion in the beginning of this section is that one can pick countably many $\left\{\Omega_{i}\right\}_{i \geqq 1}$ out of $\left\{\Omega_{c}\right\}$ such that $\bigcup_{i} \Omega_{i}$ has a full $P$ measure, and that every $\Omega_{i}$ has the following form: for $n$ outside finite set $F_{i}$ (which can be chosen once for all $\omega \in \Omega_{i}$ ), $I_{n}^{0}$ 's are exactly corresponded to the $n$ 's which belong to the connected groups of animals, or more precisely, at site $n$ we have $I_{n}^{0}$ iff $n \in G^{(i)}$ for some $i$. Furthermore, $G^{(i)}$ 's are independent of the choice $\omega \in \Omega_{i}$, and thus our decomposition fixes the position of "potential wells," although potential within them can vary (at site $n$, between 0 and $\left.|n|^{\gamma}\right)$. We will prove below that (4.2) is valid for a.e. $\omega \in \Omega_{1}$ and a.e. $E \in I$. Since our argument does not depend on the choice of $\Omega_{1}$ (it also applies to any $\Omega_{k}, k>1$, all what we use is the fixed position of "wells"), it yields the statement. In the sequel any $\omega$, unless otherwise stated, will refer to an event in $\Omega_{1}$.

Let $L>1$ be a positive constant and define a new potential $\left(C_{L}=\right.$ $\left.\left\{n: \max \left|n_{i}\right| \leqq L\right\}\right)$

$$
V_{\omega, L}= \begin{cases}V_{\omega}(n), & \text { if } n \notin C_{L} \\ L, & \text { if } n \in C_{L}\end{cases}
$$


The right choice of $L$ will circumvent technical problems with convergence of the resolvent cluster expansion constructed below. First, by making $L$ large enough, we can suppose that $C_{L} \supset F_{1}$. If

$$
H_{\omega, L}=H_{0}+V_{\omega, L}
$$

we have that $H_{\omega}$ is a finite rank, $L$-dependent perturbation of $H_{\omega, L}$. Denote by

$$
R_{\omega, L}(n, m ; z)=\left(\delta_{n},\left(H_{\omega, L}-z\right)^{-1} \delta_{m}\right)
$$

the matrix elements of the resolvent of $H_{\omega, L}$. The main technical ingredient in our proof is the following

Lemma 4.2. To every $L$ large enough we can correspond $\Omega_{L} \subset \Omega_{1}, \Omega_{L} \rightarrow \Omega_{1}$ as $L \rightarrow \infty$, such that for a.e. $\omega \in \Omega_{L}, i \in C_{L}, m \in Z^{d}$, and for every fixed $E \in I$, we have for some fixed $a>0$,

$$
\sup _{0<\varepsilon<1}\left|R_{\omega, L}(i, m ; E+i \varepsilon)\right| \leqq C_{\omega, L} \exp (-a|m|) .
$$

Lemma 4.1 yields (4.2) as follows. Since

$$
H_{\omega}-H_{\omega, L}=\sum_{i \in C_{L}} \lambda_{i}\left(\cdot, \delta_{i}\right) \delta_{i},
$$

where $\lambda_{i} \in I_{i}{ }^{0}-L$ or $I_{i}{ }^{1}-L$, we have that for $\omega \in \Omega_{L}$ and $L$ large enough

$$
R_{\omega}(n, m ; z)=R_{\omega, L}(n, m ; z)-\sum_{i \in C_{L}} \lambda_{i} R_{\omega, L}(i, m ; z) R_{\omega}(n, i ; z) .
$$

Since $R_{\omega}(n, i ; z)$ is a difference of two Herglotz functions in the upper half plane, we have that for a.e. $\omega \in \Omega_{L}$ and for a.e. $E \in I$,

$$
\max _{i \in C_{L}} \sup _{0<\varepsilon<1}\left|R_{\omega}(n, i ; E+i \varepsilon)\right| \leqq D_{n, \omega, E},
$$

where $D_{n, \omega, E}$ is a finite constant (whose dependence on $L$ is comprised within $\omega$ ). Thus, (4.2) is valid for a.e. $\omega \in \Omega_{L}$ and a.e. $E \in I$. Since $\Omega_{L} \rightarrow \Omega_{1}$ as $L \rightarrow \infty$, (4.2) follows.

It remains to prove Lemma 4.2.

4.2. Proof of Lemma 4.2. For arbitrary $X \subset Z^{d}$ denote

$$
\begin{aligned}
\partial_{\text {ext }} X & =\left\{m \in Z^{d} \backslash X:(\exists n \in X)|m-n|_{+}=1\right\}, \\
\partial_{\text {int }} X & =\left\{m \in X:\left(\exists n \in Z^{d} \backslash X\right)|m-n|_{+}=1\right\}, \\
\partial X & =\left\{(m, n): m \in \partial_{\text {ext }} X, n \in \partial_{\text {int }} X,|m-n|_{+}=1\right\} .
\end{aligned}
$$

If $H=H_{0}+V$, we say that $H_{D}$ is the operator $H$ with Dirichlet boundary condition along $\partial X$ if

$$
H_{D}-H=\sum_{(m, n) \in \partial X}\left(\cdot, \delta_{m}\right) \delta_{n}+\left(\cdot, \delta_{n}\right) \delta_{m} .
$$

$H_{D}$ is a direct sum of two operators, one on $l^{2}(X)$ and one on $l^{2}\left(Z^{d} \backslash X\right)$. The part which acts on $l^{2}(X)$ will be referred to as the restriction of $H$ to $X$. 
For $i \geqq i(L)$ all connected groups $G^{(i)}$ have empty intersection with $C_{L}$. Denote by $H_{\omega, L}^{(i)}$ the restriction of $H_{\omega, L}$ to $G^{(i)}$ for $i \geqq i(L)$, and by $R_{\omega, L}^{(i)}(n, m ; z)$ the matrix element of the $\left(H_{\omega, L}^{(i)}-z\right)^{-1}$. Let

$$
G^{(0)}=Z^{d} \backslash\left(\bigcup_{i \geqq i(L)} G^{(i)}\right) .
$$

The size of $L$ will be fixed by requirements below (recall that the statement of lemma is valid for $L$ large enough). We denote by $H_{\omega, L}^{(0)}$ and $R_{\omega, L}^{(0)}$ the restrictions of $H_{\omega, L}, R_{\omega, L}$ to $G^{(0)}$. From now on, let $n \in C_{L}, m \in Z^{d}$ be two fixed sites. For definiteness we will suppose that $m \in G^{(j)}$ for some $j \geqq i(L)$, the discussion of the case when $m \in G^{(0)}$ is analogous.

For $\operatorname{Im}(z)>2 d$ we have expansion

$$
R_{\omega, L}(n, m ; z)=-\sum_{\tau} \prod_{s \in \tau} \frac{1}{V_{\omega, L}(s)+2 d-z},
$$

where series on the right converges uniformly, and sum is over all paths connecting $n$ and $m$. By regrouping the terms in the series, we can write

$$
\begin{aligned}
R_{\omega, L}(n, m ; z)= & -\sum_{k \geqq 0} \sum_{\substack{\left(i_{1}, \ldots, i_{k}\right) \\
i_{l} \geqq i(L)}} \sum_{\substack{\left(s_{l}, t_{l}\right) \in \partial G^{\left(l_{l}\right)} \\
\left(s_{l}^{\prime}, t_{1}^{\prime}\right) \in \partial G^{\left(l_{l}\right)}}} \sum_{(s, t) \in \partial G^{(j)}} R_{\omega, L}^{(0)}\left(n, s_{1} ; z\right) \cdot R_{\omega, L}^{\left(i_{1}\right)}\left(t_{1}, t_{1}^{\prime} ; z\right) . \\
& \cdot R_{\omega, L}^{(0)}\left(s_{1}^{\prime}, s_{2} ; z\right) \cdot R_{\omega, L}^{\left(i_{2}\right)}\left(t_{2}, t_{2}^{\prime} ; z\right) \cdot R_{\omega, L}^{(0)}\left(s_{2}^{\prime}, s_{3} ; z\right) \cdot \ldots \\
& \cdots \cdot R_{\omega, L}^{\left(i_{k}\right)}\left(t_{k}, t_{k}^{\prime} ; z\right) \cdot R_{\omega, L}^{(0)}\left(s_{k}^{\prime}, s ; z\right) \cdot R_{\omega, L}^{(j)}(t, m ; z) .
\end{aligned}
$$

The above formula clearly follows from (4.6), separating the contribution of the paths within different $G^{(i)}$ 's, and using the formula analogous to the (4.6) for each $R_{\omega, L}^{(i)}$. For $i>0, j>0$ we set

$$
d(i, j)=\max \left\{|n-m| ; n \in G^{(i)}, m \in G^{(j)}\right\}, \quad d_{n}(i)=\max _{m \in G^{(i)}}|n-m| .
$$

The following lemma gives the basic estimates needed to control (4.7).

Lemma 4.3. For $E \in I, 0<\varepsilon<1$ and $L$ suffciently large:

(i) There exist random variables $A_{E}^{(i)}(\omega)$ for every $i \geqq i(L)$ such that for $t \in \partial_{\text {int }} G^{(i)}$, $t^{\prime} \in G^{(i)}$

$$
\left|R_{\omega, L}^{(i)}\left(t, t^{\prime} ; E+i \varepsilon\right)\right|<A_{E}^{(i)}(\omega) \text {. }
$$

(ii)

$$
P\left\{\omega \in \Omega_{1}: A_{E}^{(i)}(\omega)>M\right\} \leqq C / M
$$

for a uniform constant $C$ independent of $i$.

(iii) If $s \in \partial_{\mathrm{ext}} G^{(i)}$ and $s^{\prime} \in \partial_{\mathrm{ext}} G^{(j)}$ we have

$$
\begin{aligned}
& R_{\omega, L}^{(0)}\left(s^{\prime}, s ; E+i \varepsilon\right) \leqq C_{L} \exp \left(-a_{L} d(i, j)\right), \\
& R_{\omega, L}^{(0)}\left(n, s^{\prime} ; E+i \varepsilon\right) \leqq C_{L} \exp \left(-a_{L} d_{n}(j)\right) .
\end{aligned}
$$

Constants $C_{L}, a_{L}$ depend only on $L$ and furthermore $C_{L} \rightarrow 0, a_{L} \rightarrow \infty$ as $L \rightarrow \infty$. 
Proof. Let $H=H_{0}+V$ be a discrete Schrödinger operator, and let $H_{D}$ be its restriction to a set $X \subset Z^{d}$. If $\delta=\operatorname{dist}\left(z, \sigma\left(H_{D}\right)\right.$ ), it is a standard result (see e.g. [2], Exercise III.6.8) that for $m, n \in X$ we have

$$
\left|R_{D}(m, n ; z)\right| \leqq \frac{2}{\delta} \exp (-\ln (1+\delta / 4 d)|m-n|) .
$$

Defining

$$
A_{E}^{(i)}(\omega)=2 / \operatorname{dist}\left(E, \sigma\left(H_{\omega, L}^{(i)}\right)\right),
$$

we observe that part (i) follows from (4.8). Part (ii) is an immediate consequence of Propositions VIII.1.8 and VIII.4.8 in [2]. Part (iii) follows from (4.8) and observation that for $L$ large enough

$$
\operatorname{dist}\left(E, \sigma\left(H_{\omega, L}^{0}\right)\right) \geqq L^{\gamma}-E_{0}-4 d .
$$

The sets $\Omega_{L}$ are defined as

$$
\Omega_{L}=\left\{\omega \in \Omega_{1} ; A_{E}^{(i)}(\omega) \leqq\left|n_{i}\right|^{d+1} \text { for } i \geqq i(L)\right\} .
$$

Part (ii) of the above lemma yields

$$
P\left(\Omega_{L}\right) \geqq P\left(\Omega_{1}\right)-\sum_{i \geqq i(L)} C /\left|n_{i}\right|^{d+1}=P\left(\Omega_{1}\right)-O(1 / i(L)) .
$$

Since $i(L) \rightarrow \infty$ as $L \rightarrow \infty$ sets $\Omega_{L}$ are as required by Lemma 4.1. For $L$ large enough, $\omega \in \Omega_{L}, E \in I$ and for $1>\varepsilon>0$ we have

$$
\left|R_{\omega, L}(n, m ; E+i \varepsilon)\right|<C_{L} \exp \left[-\left(a_{L} / 2\right) \cdot|n-m|\right] \sum_{n \geqq 0}\left(\alpha_{L}\right)^{n},
$$

where

$$
\begin{aligned}
\alpha_{L}=\sup _{i \geqq i(L)} \sup _{s \in \partial_{\text {ext }} G^{(i)}}\left|n_{i}\right|^{d+1} \sum_{j \geqq i(L)} \sup _{\substack{\left.s_{1}, t_{1}\right) \in \partial G^{(j)} \\
t_{2} \in \partial_{\text {in }} G^{(j)}}} \mid R_{\omega, L}^{(0)}\left(s, s_{1} ; E+i \varepsilon\right) \\
\\
\left.\cdot R_{\omega, L}^{(j)}\left(t_{1}, t_{2} ; E+i \varepsilon\right)\right|^{1 / 2} .
\end{aligned}
$$

In (4.9) $C_{L} \rightarrow 0$ as $L \rightarrow \infty$. One can easily estimate

$$
\alpha_{L} \leqq \sup _{i \geqq i(L)} C\left|n_{i}\right|^{d+1} \exp \left(-D\left|n_{i}\right|\right),
$$

where constants $C$ and $D$ are uniform. For $L$ large enough, $\alpha_{L}<1$, and statement follows from (4.10).

4.2. Discrete Spectrum is Finite. To prove that for a.e. $\omega$ the number of eigenvalues lying in $\left[0, a_{k}\right)$ is finite, in the notation of Lemma 4.1 , it suffices to show that to every $L$ large enough we can correspond $\Omega_{L} \subset \Omega_{1}, \bigcup \Omega_{L}=\Omega_{1}$, such that for $\omega \in \Omega_{L}$ $H_{\omega, L}$ has no spectrum in $\left[0, a_{k}\right)$ (since $H_{\omega, L}-H_{\omega}$ is an operator of rank $(2 L+1)^{d}$, we then have $\left.\sigma_{\text {disc }}\left(H_{\omega}\right) \leqq(2 L+1)^{d}\right)$.

$H_{\omega, L}$ has no eigenvalues in $\left[0, a_{k}\right)$ if for all $n, m \in Z^{d}$,

$$
\sup _{E<a_{k}} \sup _{\varepsilon>0}\left|R_{\omega, L}(n, m ; E+i \varepsilon)\right|<\infty \text {. }
$$


We write the cluster expansion (4.7) of $R_{\omega, L}$, and estimate for $E<a_{k}$

$$
\left|R_{\omega, L}^{(i)}\left(t, t^{\prime} ; E+i \varepsilon\right)\right| \leqq 2 / \operatorname{dist}\left(a_{k}, \sigma\left(H_{\omega, L}^{(i)}\right)\right)=A_{a_{k}}^{(i)}(\omega)
$$

The rest of the argument follows line by line that of the previous section.

\section{Proof of Theorem 1.4}

We will prove Theorem 1.4 in the case $d=1$, the argument being essentially the same for $d>1$. For a purpose of this section it is convenient to redefine $H_{D}$ (operator with Dirichlet boundary condition) by subtracting $\left(\cdot, \delta_{n}\right) \delta_{n}+\left(\cdot, \delta_{m}\right) \delta_{m}$ from (4.5), ensuring that $H \leqq H_{D}$. For $L$ a positive integer, let $C_{L}$ be an interval of size $2 L$ centered at the origin, and let $H_{\omega, L}$ be the restriction of $H_{\omega}$ to $C_{L}$. We suppose that $1 / k>\alpha>1 /(k+1)$, and that $E \in\left(a_{j}, a_{j-1}\right)\left(a_{0}=\infty\right)$ for some $1 \leqq j \leqq k$. The case $\alpha=1 / k$, we treat separately.

Let $0<\delta<1-j \alpha$, and for fixed $\omega$ suppose that $C_{L, \delta}=C_{\left[L^{\delta}\right]+1} \supset A(0)$ (see (4.1), $[\cdot]$ stands for the greatest integer part). Define $H_{\omega, L}^{\delta}$ from $H_{\omega, L}$ by setting the potential to be of the size $L$ within $C_{L, \delta}$. In the sequel $N_{E}(H)$ stands for the number of eigenvalues of an operator $H$ which are less than $E$. Since $\left|N_{E}\left(H_{\omega, L}\right)-N_{E}\left(H_{\omega, L}^{\delta}\right)\right|=O\left(L^{\delta}\right)$, it suffices to prove the statement for $N_{E}\left(H_{\omega, L}^{\delta}\right)$. We numerate the connected group of animals by their distance from the origin, and set

$$
Z_{L}^{(i)}(\omega)=G^{(i)}(\omega) \cap\left(C_{L} \backslash C_{L, \delta}\right), \quad Z_{L}(\omega)=\bigcup Z_{L}^{(i)}(\omega), \quad Z_{L}^{(0)}(\omega)=C_{L} \backslash Z_{L}(\omega)
$$

Let $\hat{H}_{L, \omega}$ be the operator obtained from $H_{\omega, L}$ by putting the Dirichlet boundary condition along $\partial Z(\omega)$.

Lemma 5.1. There exist $\varepsilon>0$ such that for a.e. $\omega$,

$$
\begin{array}{r}
\liminf _{L \rightarrow \infty} \frac{N_{E}\left(H_{\omega, L}^{\delta}\right)}{L^{1-j \alpha}} \geqq \liminf _{L \rightarrow \infty} \frac{N_{\left(E-1 / L^{\varepsilon}\right)}\left(\hat{H}_{\omega, L}\right)}{L^{1-j \alpha}}, \\
\underset{L \rightarrow \infty}{\limsup } \frac{N_{\left(E+1 / L^{\varepsilon}\right)}\left(\hat{H}_{\omega, L}\right)}{L^{1-j \alpha}} \geqq \limsup _{L \rightarrow \infty} \frac{N_{E}\left(H_{\omega, L}^{\delta}\right)}{L^{1-j \alpha}} .
\end{array}
$$

Proof. (5.1) is immediate since $\hat{H}_{\omega, L} \geqq H_{\omega, L}^{\delta}$. To prove (5.2) we proceed as follows. Let $R(n, m), \hat{R}(n, m)$ be matrix elements of $R=\left(H_{\omega, L}+1\right)^{-1}, \hat{R}=\left(\hat{H}_{\omega, L}+1\right)^{-1}$. First, we have that

$$
|R(n, m)-\hat{R}(n, m)| \leqq \frac{C}{L^{\gamma \delta}} \exp (-a|n-m|)
$$

for uniform constants $C$ and $a\left(\gamma\right.$ is as in (2.8)). We will prove (5.2) in the case $n \in Z^{(i)}$, $m \in Z^{(i)}$, the other cases having similar proofs. We have

$$
R(n, m)=\hat{R}(n, m)+\sum_{(s, t) \in \partial Z^{(i)}} \sum_{\left(s^{\prime}, t^{\prime}\right) \in \partial Z^{(i)}} \hat{R}(n, s) \hat{R}\left(t, s^{\prime}\right) R\left(t^{\prime}, m\right) .
$$


Using (4.8) we estimate

$$
\begin{gathered}
\left|\hat{R}\left(t, s^{\prime}\right)\right| \leqq \frac{C}{L^{\gamma \delta}} \exp \left(-a\left|t-s^{\prime}\right|\right), \\
|\hat{R}(n, s)| \leqq C \exp (-a|n-s|), \quad\left|R\left(t^{\prime}, m\right)\right| \leqq C \exp \left(-a\left|t^{\prime}-m\right|\right),
\end{gathered}
$$

where $C$ and $a$ are uniform constants, and (5.3) follows.

$\hat{R}$ splits into direct sum of operators (after eventually renumerating $Z^{(i)}$ 's)

$$
\hat{R}=\bigoplus_{i=1}^{l} \hat{R}^{(i)} \oplus \hat{R}^{(0)} \text { acting on } \bigoplus_{i=1}^{l} l^{2}\left(Z^{(i)}\right) \oplus l^{2}\left(Z^{(0)}\right) \text {. }
$$

By permuting the basis of $l^{2}\left(C_{L}\right)$ we can write $\hat{R}$ in the matrix form

$$
\hat{R}=\left(\begin{array}{cccc}
\hat{R}^{(1)} & 0 & \cdots & 0 \\
0 & \hat{R}^{(2)} & \cdots & 0 \\
\vdots & \vdots & \ddots & \vdots \\
0 & 0 & \cdots & \hat{R}^{(0)}
\end{array}\right)
$$

For $1 \leqq i \leqq l, \hat{R}^{(i)}$ is a matrix of dimension $k_{i} \times k_{i}$ for some $k_{i} \leqq k$. Let $\tilde{R}$ be the operator $R$ in permuted basis, and let $\left(k_{0}=0\right)$

$$
\rho_{i}=\max _{k_{i-1} \leqq n \leqq k_{i}} \sum_{m}\left|\hat{R}^{(i)}(n, m)-\tilde{R}(n, m)\right| .
$$

It follows from (5.3) that $\rho_{i} \leqq C / L^{\gamma \delta}$ for uniform constant $C$.

Let $1 /(\lambda+1)$ be an eigenvalue of $R$ and let $\phi$ be corresponding eigenvector. If $\max _{n \in C_{L}}|\phi(n)|$ is achieved for some $n \in Z^{(i)}, i>0$, then proceeding as in the proof of Gershorgin's theorem [21], one can show that there exist eigenvalue $E_{j}^{(i)}$ of $\hat{R}^{(i)}$ such that

$$
\left|E_{j}^{(i)}-\lambda\right| \leqq C / L^{\gamma \delta}
$$

If $\max |\phi(n)|$ is achieved for $n \in Z^{(0)}$, it is elementary to show that $\lambda \geqq c L^{\gamma}$ for some $c>0$. Thus, for $L$ large enough, the eigenvalues of $H_{\omega, L}^{\delta}$ which are less than $E$ are on the distance $C / L^{\gamma \delta}$ from the eigenvalues of $\hat{H}_{\omega, L}$ which are less than $E$, and statement follows.

The probabilistic part of the proof goes as follows. Let $H^{(i)}$ be the restriction of $\hat{H}_{\omega, L}$ to $Z^{(i)}$ and let $\chi_{n}$ be random variables defined on the following way:

$$
\chi_{n}(\omega)= \begin{cases}N_{E}\left(H^{(i)}\right), & \text { if } n \in Z^{(i)} \text { and } d\left(Z^{(i)}, 0\right)=|n| \\ 0, & \text { otherwise }\end{cases}
$$

$\chi_{n}$ are independent random variables for $L$ large enough.

Lemma 5.2. If $n \in C_{\left[L^{\delta}\right]+j}$ or ||$n|-L|<j, \mathbf{E}\left(\chi_{n}(\omega)\right)=0$. Otherwise, there exist constant $\varepsilon(L), \varepsilon(L) \rightarrow 0$ as $L \rightarrow \infty$, such that

$$
(1-\varepsilon(L)) \frac{N_{j}(E)}{|n|^{j \alpha}} \leqq \mathbf{E}\left(\chi_{n}\right) \leqq(1+\varepsilon(L)) \frac{N_{j}(E)}{|n|^{j \alpha}},
$$

where $N_{j}(E)$ is a continuous function on $\left(a_{j}, a_{j-1}\right)$. Furthermore, $N_{j}(E) \sim$ $D_{j}\left(E-a_{j}\right)^{j}$ as $E \rightarrow a_{j}$ for a suitable constant $D_{j}$. 
Proof. To show that $\mathbf{E}\left(\chi_{n}\right)=0$ under the conditions $|n| \leqq\left[L^{\delta}\right]+j$ or ||$n|-L|<j$ is trivial. To prove (5.5), note that $N_{E}\left(H^{(i)}\right)=0$ unless $Z^{(i)}$ contains an animal of the size $j$ or bigger. Let $A$ be the operator on $l$-dimensional space $(l \geqq j)$ defined by

$$
A\left(\lambda_{1}, \lambda_{2}, \ldots, \lambda_{l}\right)=H_{0}+\sum_{i=1}^{l} \lambda_{i}\left(\cdot, \delta_{i}\right) \delta_{i},
$$

where $H_{0}$ is a finite dimensional Laplacian (see (1.3)). Ordering eigenvalues of $A$ in the increasing order,

$$
E_{1}\left(\lambda_{1}, \lambda_{2}, \ldots, \lambda_{l}\right)<E_{2}\left(\lambda_{1}, \lambda_{2}, \ldots, \lambda_{l}\right) \leqq \ldots \leqq E_{l}\left(\lambda_{1}, \lambda_{2}, \ldots, \lambda_{l}\right),
$$

we observe that $E_{i}$ are continuous functions of $\left(\lambda_{1}, \lambda_{2}, \ldots, \lambda_{l}\right)$, and if any $\lambda_{i}$ is large enough, $E_{1}>E[12]$. If $A_{p}=\left\{\left(\lambda_{1}, \ldots, \lambda_{l}\right): N_{E}(A)=p\right\}$, then $A_{p}, 1 \leqq p \leqq l$, are disjoint bounded measurable sets. The estimates (5.5) are now obvious (we remark that $\varepsilon(L)$ part contains contribution from the animals within $Z^{(i)}$ of the size strictly bigger than $j$, if any). The continuity of function $N_{j}(E)$ is a consequence of Proposition VIII.4.10 in [2].

Finally, let $\phi$ be the ground state corresponded to the eigenvalue $a_{j}$. The regular perturbation theory [17] yields that

$$
E_{1}\left(\lambda_{1}, \lambda_{2}, \ldots, \lambda_{j}\right)=a_{j}+\sum \lambda_{i}|\phi(n)|^{2}+O\left(\sum\left|\lambda_{i}\right|^{2}\right) .
$$

Furthermore, for $E-a_{j}$ small enough we have

$$
N_{j}(E)=\mu\left\{\left(\lambda_{1}, \lambda_{2}, \ldots, \lambda_{j}\right): \sum|\phi(i)|^{2} \lambda_{i}+O\left(\sum\left|\lambda_{i}\right|^{2}\right)<E-a_{j}\right\},
$$

where $\mu$ stands for a Labesgue measure. It is now elementary to show that as $E \rightarrow a_{j}$,

$$
N_{j}(E) \sim D_{j}\left(E-a_{j}\right)^{j}, \quad D_{j}=\frac{1}{j ! \prod|\phi(i)|^{2}} .
$$

Remark. For $d>1$, the constants $D_{j}$ have a more complicated form, and are intrinsically of combinatorial nature.

Since $N_{E}\left(\hat{H}_{\omega, L}\right)=\sum_{n \in C_{L}} \chi_{n}(\omega)$, we have

$$
\begin{aligned}
\lim _{L \rightarrow \infty} \frac{\mathbf{E}\left(N_{E}\left(\hat{H}_{\omega, L}\right)\right)}{L^{1-j \alpha}} & =\lim _{L \rightarrow \infty} \sum_{n \in C_{L}} \frac{\mathbf{E}\left(\chi_{n}(\omega)\right)}{L^{1-j \alpha}} \\
& =2 \int_{0}^{1} \frac{N_{j}(E)}{|x|^{j \alpha}} d x \\
& =\frac{2}{1-j \alpha} N_{j}(E) .
\end{aligned}
$$

It remains to show that

$$
\lim _{L \rightarrow \infty} \frac{\mathbf{E}\left(N_{E}\left(\hat{H}_{\omega, L}\right)\right)-N_{E}\left(\hat{H}_{\omega, L}\right)}{L^{1-j \alpha}}=0 \text { for a.e. } \omega .
$$


Let $\eta_{n}(\omega)=\chi_{n}(\omega)-\mathbf{E}\left(\chi_{n}(\omega)\right)$. Then, if $p$ is a positive integer, we get as in (2.4)

$$
\begin{aligned}
\mathbf{E}\left(\sum_{n \in C_{L}} \eta_{n}(\omega)\right)^{2 p} & \leqq C \mathbf{E}\left(\sum_{n \in C_{L}} \chi_{n}(\omega)\right)^{p} \\
& \leqq C L^{p(1-j \alpha)}
\end{aligned}
$$

where constant $C$ does not depend on $L$. Defining

$$
A_{L}(\omega)=\left|\mathbf{E}\left(N_{E}\left(\hat{H}_{\omega, L}\right)\right)-N_{E}\left(H_{\omega, L}\right)\right|,
$$

we have as in Sect. 2.1,

$$
\begin{aligned}
P\left\{\omega:(2 L)^{j \alpha-1} A_{L}(\omega) \geqq \varepsilon\right\} & \leqq \frac{C}{\varepsilon^{2 p}} L^{2 p(j \alpha-1)} \cdot L^{p(1-j \alpha)} \\
& \leqq \frac{C}{\varepsilon^{2 p}} L^{-p(1-j \alpha)}
\end{aligned}
$$

For $p$ large enough, $p(1-j \alpha)>1$, and the Borel-Cantelli Lemma yields (5.6).

When $\alpha=1 / k$ and $E \in\left(a_{j}, a_{j-1}\right), j>k$, the above argument applies without changes. The case $\alpha=1 / k, E \in\left(a_{k}, a_{k-1}\right)$, is special, and to prove (1.9) we argue as follows. In the construction in the beginning of section interval $C_{\left[L^{\delta}\right]+1}$ is replaced by an interval $C_{\left[(\ln L)^{\delta}\right]+1}$ for $\delta$ small enough. Lemma 5.1 is valid after replacement of $L^{\varepsilon}$ with $(\ln L)^{\varepsilon}$, and Lemma 5.2 is valid as it stands. Thus

$$
\lim _{L \rightarrow \infty} \frac{\mathbf{E}\left(N_{E}\left(\hat{H}_{\omega, L}\right)\right)}{\ln L}=2 N_{k}(E) .
$$

It remains to show that for a.e. $\omega$

$$
\lim _{L \rightarrow \infty} \frac{A_{L}(\omega)}{\ln L}=0
$$

with $A_{L}(\omega)$ given by (5.6). First, one can show that

$$
\mathbf{E}\left(\left(A_{L}(\omega)\right)^{2 p}\right) \leqq C^{p}(\ln L)^{p},
$$

where $C$ is a uniform constant independent of $p$ and $L$. Thus

$$
\mathbf{E}\left(\exp \left(A_{L}(\omega)^{2}\right)\right) \leqq \sum_{k} \frac{\mathbf{E}\left(A_{L}(\omega)^{2 k}\right)}{k !} \leqq L^{C^{2}}
$$

We have

$$
\begin{aligned}
P\left\{\omega:(\ln L)^{-1} A_{L}(\omega) \geqq \varepsilon\right\} & \leqq P\left\{\omega: \exp \left(A_{L}(\omega) / \varepsilon\right)^{2} \geqq \exp \left((\ln L)^{2}\right)\right\} \\
& \leqq \frac{1}{\exp \left((\ln L)^{2}\right)} \mathbf{E}\left(\exp \left(A_{L}(\omega) / \varepsilon^{2}\right)\right) \\
& \leqq \frac{L^{(C / \varepsilon)^{2}}}{L^{\ln L}}
\end{aligned}
$$

and the Borel-Cantelli Lemma again yields the statement. 


\section{Appendix}

Proof of Theorem 2.2. For simplicity of notation we set $n_{0}=0$. It suffices to prove that under the conditions of theorem, (2.12) is valid for a.e. $E \in(a, b)$ where $(a, b)$ is a fixed interval of finite size. We introduce boundary blocks $B_{k}$ and main blocks $M_{k}$ as $B_{0}=\emptyset, M_{0}=\emptyset$,

$$
B_{k}=\Gamma_{k}^{+} \backslash \Gamma_{k}^{-}, \quad M_{k}=\left(\Gamma_{k}^{+} \backslash M_{k-1}\right) \cup B_{k-1} .
$$

The restrictions of $H$ to $M_{k}, B_{k}$ we denote by $H_{M_{k}}, H_{B_{k}}$, and by $R_{M_{k}}(n, m ; z)$, $R_{B_{k}}(n, m ; z)$ the kernels of $\left(H_{M_{k}}-z\right)^{-1},\left(H_{B_{k}}-z\right)^{-1}$. For $m \in Z^{d}$, let $M_{k}$ be the main block such that $m \in M_{k}, m \notin M_{k+1}$. Let's suppose that $m \in M_{k} \backslash B_{k-1}$, the treatment of the case $m \in B_{k-1}$ is analogous. Our first goal is to obtain a suitable cluster expansion with respect to the blocks $M_{k}, B_{k}$. For $\operatorname{Im}(z)>2 d$ we have

$$
R(0, m ; z)=-\sum_{\tau} \prod_{s \in \tau} \frac{1}{V(s)+2 d-z},
$$

where summation is over all paths $\tau$ connecting 0 and $m$. We will divide set of all such paths into disjoint classes on the following way. Let $\Gamma$ be graph whose vertices are positive integers and whose bonds connect only successive integers. To every path $\tau$ in $Z^{d}$ connecting 0 and $m$ we correspond a unique path $\gamma$ on $\Gamma$ connecting 0 and $k$ on the following way: $\gamma$ starts at 0 and whenever $\tau$ leaves $M_{i}$ and crosses to $M_{i+1} \backslash M_{i}$ or $M_{i-1} \backslash M_{i}, \gamma$ jumps from vertex $i$ to respectively vertex $i+1$ or $i-1$. Thus, the set of all paths connecting 0 and $m$ can be divided into disjoint classes, associated to the paths on $\Gamma$ connecting 0 and $k$. We denote by $\gamma$ the class corresponding to $\gamma$.

For any $j$, the boundary $\partial B_{j}$ can be divided into two parts, the inner boundary

$$
\partial_{\text {in }} B_{j}=\left\{(n, m):(n, m) \in \partial B_{j}, m \in \Gamma_{j}^{-}\right\},
$$

and outer boundary $\partial_{\text {out }} B_{j}=\partial B_{j} \backslash \partial_{\text {in }} B_{j}$. If path $\gamma$ is given by $\left(1, i_{1}, i_{2}, \ldots, i_{l}, k\right)$ (in fact, one has $i_{1}=2$ unless the path is of length 1 ), we define a new sequence $\left(1, j_{1}, j_{2}, \ldots, j_{l}\right)$ such that

$$
j_{k}= \begin{cases}i_{s}, & \text { if } i_{s+1}>i_{s} \\ i_{s-1}, & \text { otherwise }\end{cases}
$$

and a sequence of boundaries $L_{1}=\partial_{\text {in }} B_{1}, L_{1}^{\prime}=\partial_{\text {out }} B_{1}$,

$$
\begin{aligned}
L_{j_{s}} & = \begin{cases}\partial_{\text {in }} B_{i_{s}}, & \text { if } i_{s+1}>i_{s} ; \\
\partial_{\text {out }} B_{i_{s-1}}, & \text { otherwise },\end{cases} \\
L_{j_{s}}^{\prime} & = \begin{cases}\partial_{\text {out }} B_{i_{s}}, & \text { if } i_{s+1}>i_{s} ; \\
\partial_{\text {int }} B_{i_{s-1}} & \text { otherwise }\end{cases}
\end{aligned}
$$

If

$$
R^{(\gamma)}(0, m ; z)=-\sum_{\tau \in(\gamma)} \prod_{s \in \tau} \frac{1}{V(s)+2 d-z},
$$


we can write

$$
\begin{aligned}
R^{(\gamma)}(0, m ; z)= & \sum_{\substack{\left(t_{1}, s_{1}\right) \in \partial L_{1} \\
\left(t_{1}^{\prime}, s_{1}^{\prime}\right) \in \partial L_{1}^{\prime}}} \sum_{\begin{array}{c}
\left(t_{i^{\prime}}, s_{i^{\prime}}\right) \in \partial L_{i_{j}}^{\prime} \\
\left.t_{i_{j}}^{\prime}, i_{i}^{\prime}\right) \in \partial L_{i_{j}}^{\prime} \\
1 \leqq j \leqq l
\end{array}} R_{M_{1}}\left(0, t_{1} ; z\right) \cdot R_{B_{1}}\left(s_{1}, s_{1}^{\prime}, z\right) R_{M_{i_{1}}}\left(t_{1}^{\prime}, t_{i_{1}} ; z\right) . \\
& \cdot R_{B_{i_{1}}}\left(s_{i_{1}}, s_{i_{1}}^{\prime} ; z\right) \cdot \ldots \cdot R_{M_{i_{l}}}\left(t_{i_{l}-1}^{\prime}, t_{i_{l}}\right) R_{B_{i_{l}}}\left(s_{i_{l}}, s_{i_{l}}^{\prime}\right) R_{M_{k}}\left(t_{i_{l}}^{\prime}, m\right) .
\end{aligned}
$$

(If $m=0$ the term $1 /(V(0)+2 d-z)$ ) should be added.) The strategy of controlling $R^{(\gamma)}$ is now clear: the resolvents of boundary blocks are small and will compensate for the size of resolvents of main blocks. The following lemma, proven in [15], is the main technical ingredient in establishing (2.12). We give proof for readers convenience.

Lemma A.1. Let $M_{k}$ be arbitrary main block.

(i) For any $L>0$ and any $n, m \in M_{k}$,

$$
\mu\left\{E \in(a, b) ;\left|R_{M_{k}}(n, m ; E)\right|>L\right\} \leqq C / L,
$$

where $\mu$ is a Lebesgue measure and $C$ a uniform constant.

(ii) For any $n \in M_{k}$,

$$
\mu\left\{E \in(a, b): \sum_{m \in M_{k}}\left|R_{M_{k}}(n, m ; E)\right|^{2}>L\right\} \leqq 4\left(\# M_{k} / L\right)^{1 / 2} .
$$

Proof. (i) Let $L_{k}=\# M_{k}$. Then

$$
R_{M_{k}}(n, m ; E)=\sum_{i=1}^{L_{k}} \frac{\phi_{i}(n) \phi_{i}(m)}{E-E_{i}},
$$

where $\phi_{i}$ are eigenfunctions and $E_{i}$ eigenvalues of $H_{M_{k}}$. We can write

$$
R_{M_{k}}(n, m ; E)=\int_{R} \frac{d v(\lambda)}{E-\lambda},
$$

where measure $v$ has atoms $\phi_{i}(n) \phi_{i}(m)$ at points $E_{i}$. Since

$$
\begin{aligned}
\sum_{i=1}^{L_{k}}\left|\phi_{n}\right| \cdot\left|\phi_{m}\right| & \leqq\left(\sum_{i=1}^{L_{k}}\left|\phi_{i}(n)\right|^{2}\right)^{1 / 2} \cdot\left(\sum_{i=1}^{L_{k}}\left|\phi_{i}(m)\right|^{2}\right)^{1 / 2} \\
& \leqq\left\|\delta_{n}\right\| \cdot\left\|\delta_{n}\right\|=1,
\end{aligned}
$$

we have $|\mu|_{\text {var }} \leqq 1$, and (i) follows from Kolmogorov's theorem (see [15] for an elementary proof due to T. Wolff).

(ii) Let

$$
a(E)=\sum_{m \in M_{k}}\left|R_{M_{k}}(n, m ; E)\right|^{2}=\sum_{i=1}^{L_{k}} \frac{\left|\phi_{i}(n)\right|^{2}}{\left|E-E_{i}\right|^{2}},
$$

and let

$$
\Delta_{i}=\left\{E \in(a, b):\left|E-E_{i}\right| \leqq\left|\phi_{i}(n)\right| / L^{1 / 2}\right\}
$$


First, we have that

$$
\sum_{i=1}^{L_{k}} \mu\left(\Delta_{i}\right)=2 L^{-1 / 2} \sum_{i=1}^{L_{k}}\left|\phi_{i}(n)\right| \leqq 2 \cdot\left(L_{k} / L\right)^{1 / 2}
$$

On the other hand

$$
\begin{aligned}
\int_{R \backslash\left(S_{i}\right)} a(E) d E & \leqq \sum_{i=1}^{L_{k}} \int_{\left(\left|\phi_{l}(n)\right| / L\right)^{1 / 2}} \frac{\left|\phi_{i}(n)\right|^{2}}{E^{2}} d E \\
& \leqq \sum_{i=1}^{L_{k}} 2 L^{1 / 2}\left|\phi_{i}(n)\right| \\
& \leqq 2 \cdot\left(L \cdot L_{k}\right)^{1 / 2}
\end{aligned}
$$

Chebyshev's inequality yields

$$
\mu\left\{E \in R \backslash\left(\bigcup \Delta_{i}\right): a(E)>L\right\} \leqq \frac{1}{L} \int_{R \backslash\left(\Delta_{t}\right)} a(E) d E=2\left(L_{k} / L\right)^{1 / 2}
$$

and result follows.

We now finish the proof of Theorem 2.2 as follows. Equation (2.11) implies that for $E \in(a, b)$ and for $j$ large enough,

$$
\left|R_{B_{j}}(n, m ; E)\right| \leqq C D_{j}^{-(d-1)} j^{-(1+\delta)},
$$

where $C$ is a uniform constant. Without loss of generality we can assume that (A.1) holds for $j \geqq 1$. The part (i) of Lemma A.1 yields that for $\varepsilon>0$,

$$
\sum_{j} \mu\left\{E \in(a, b): \max _{n, m \in M_{j}}\left|R_{M_{j}}(n, m ; E)\right|>\varepsilon j^{1+\delta}\right\} \leqq(C / \varepsilon) \sum j^{-(1+\delta)}<\infty .
$$

Thus, for a.e. $E \in(a, b)$ and for $j \geqq j(E)$,

$$
\max _{n, m \in M_{j}}\left|R_{M_{j}}(n, m ; E)\right| \leqq \varepsilon j^{1+\delta} .
$$

Without loss of generality we can assume that $j(E)=1$.

We now have that for any $\varepsilon>0$

$$
\left|R^{(\gamma)}(0, m ; E+i \varepsilon)\right| \leqq|C \varepsilon|^{|\gamma|} \sum_{(t, s) \in \partial L_{j_{s}}^{\prime}}\left|R_{M_{k}}(t, m ; E)\right|
$$

where $C$ is a uniform constant and $|\gamma|$ is the length of path $\gamma$. Thus,

$$
|R(0, m ; E+i \varepsilon)|^{2} \leqq \sum_{\gamma} \# \partial B_{k}|C \varepsilon|^{|\gamma|} \sum_{(t, s) \in \partial L_{j_{s}}^{\prime}}\left|R_{M_{k}}(t, m ; E)\right|^{2},
$$

where summation is over all paths $\gamma$ on $\Gamma$ connecting 0 and $k$. The inequality (A.2) is derived under the condition that $m \in M_{k} \backslash B_{k-1}$. When $m \in B_{k-1}$, arguing on an analogous way, one can derive (A.2) (some care is required since path can connect 0 and $m$ without leaving $M_{k-1}$, but with right conventions such ambiguities are easily resolved). If $C \varepsilon<1 / 2$ it is elementary to show that

$$
\sum_{\gamma}(C \varepsilon)^{|\gamma|} \leqq C_{1}(C \varepsilon)^{k}
$$


where $C_{1}$ is uniform constant. Thus

$$
\begin{aligned}
\sum_{m \in M_{k}}|R(0, m ; E+i \varepsilon)|^{2} & \leqq \# \partial B_{k} C_{1}^{2}(C \varepsilon)^{2 k} \sum_{m \in M_{k}} \sum_{(t, s) \in \partial L_{j_{s}}^{\prime}} R_{M_{k}}(t, m ; E) \\
& \leqq\left(\# \partial B_{k}\right)^{2} C_{1}^{2}(C \varepsilon)^{2 k} \max _{t} \sum_{m \in M_{k}}\left|R_{M_{k}}(t, m ; E)\right|^{2} .
\end{aligned}
$$

Since $\# M_{j} \leqq C D_{j}^{d}$ for uniform $C$, part (ii) of Lemma A.1 yields

$$
\mu\left\{E \in(a, b): \max _{t} \sum_{m \in M_{j}}\left|R_{M_{j}}(t, m ; E)\right|^{2}>D_{j}^{d} j^{2(1+\delta)}\right\} \leqq 4 / j^{1+\delta},
$$

and thus for a.e. $E \in(a, b)$ and for $j \geqq j(E)$,

$$
\max _{t \in M_{j}} \sum_{m \in M_{j}}\left|R_{M_{j}}(t, m ; E)\right|^{2} \leqq D_{j}^{d} j^{2(1+\delta)} .
$$

Again, without loss of generality we can assume $j(E)=1$. Then, using that $D_{k} \leqq c^{k}$, we can derive that for a.e. $E \in(a, b)$ and $\varepsilon$ small enough

$$
\sum_{m \in M_{k}}|R(0, m ; E+i \varepsilon)|^{2} \leqq C_{1}^{2}\left(C_{2} \varepsilon\right)^{2 k},
$$

where $C_{1}, C_{2}$ are uniform constants. Choosing $\varepsilon$ small enough, we obtain that for a.e. $E \in(a, b)$ there exists $\alpha, 0<\alpha<1$, such that

$$
\sum_{m \in M_{k}}|R(0, m ; E+i \varepsilon)|^{2} \leqq \alpha^{2 k}
$$

Consequently, for $\varepsilon$ small enough

$$
\sum_{m \in Z^{d}}|R(0, m ; E+i \varepsilon)|^{2} \leqq \sum_{k} \sum_{m \in M_{k}}|R(0, m ; E+i \varepsilon)|^{2} \leqq \sum_{k} \alpha^{2 k}<\infty
$$

and result follows.

\section{References}

1. Bilingsley, P.: Convergence of Probability Measures. New York: John Wiley and Sons 1968

2. Carmona, R., Lacroix, J.: Spectral theory of Random Schrödinger operators. Boston: Birkhauser 1990

3. Chavel, I.: Eigenvalues in Riemannian Geometry. New York: Academic Press 1984

4. Cycon, H., Froese, R., Kirsch, W., Simon, B.: Schrödinger Operators. Berlin, Heidelberg, New York: Springer 1987

5. Deylon, F.: Appearance of purely singular continuous spectrum in a class of random Schrödinger operators. J. Stat. Phys. 40, 621 (1985)

6. Delyon, F., Levy, Y., Souillard, B.: Anderson localization for multidimensional systems at large disorder or low energy. Commun. Math. Phys. 100, 463 (1985)

7. Deylon, F., Simon, B., Souillard, B.: From power-localized to extended states in a class of one-dimensional disordered systems. Phys. Rev. Lett. 52, 2187 (1985)

8. Deylon, F., Simon, B., Souillard, B.: From power pure point to continuous spectrum in disordered systems. Ann. Inst. H. Poincaré 42, 283 (1985)

9. Gordon, A.Y., Molchanov, S.A., Tsagani, B.: Spectral theory of one-dimensional Schrödinger operators with strongly fluctuating potential. Funct. Anal. Appl. 25, 236 (1992)

10. Grimmet, G.: Percolation. Berlin, Heidelberg, New York: Springer 1989

11. Jakšić, V.: Ph.D. thesis, Caltech 1992

12. Kato, T.: Perturbation Theory for Linear Operators. Berlin, Heidelberg, New York: Springer 1966 
13. Kirsch, W., Kotani, S., Simon, B.: Absence of absolutely continuous spectrum for onedimensional random but deterministic Schrödinger operators. Ann. Inst. H. Poincaré 42, 383 (1985)

14. Kirsch, W., Molchanov, S., Pastur, L.: One-dimensional Schrödinger operator with unbounded potential. Funct. Anal. Prilozhen. 24, 14 (1990)

15. Molchanov, S.: Lectures given at Caltech, Spring 1990

16. Molchanov, S.: Localization and intermittency: New results. Talk given at Internatonal Congress of Mathematics, Kyoto 1990

17. Reed, M., Simon, B.: Methods of Modern Mathematical Physics, IV. Analysis of Operators. London: Academic Press 1978

18. Simon, B.: Some Jacobi matrices with decaying potential and dense point spectrum. Commun. Math. Phys. 87, 253 (1982)

19. Simon, B., Spencer, T.: Trace class perturbations and the absence of absolutely continuous spectrum. Commun. Math. Phys. 125, 113 (1989)

20. Simon, B., Wolff, T.: Singular continuous spectrum under rank one perturbations and localization for random Hamiltonian. Commun. Pure Appl. Math. 39, 75 (1986)

21. Friedberg, S.H., Arnold, J.I., Lawrence, E.S.: Linear Algebra, ed. Englewood Cliffs, NJ: Prentice Hall 1989

Communicated by T. Spencer 